\title{
Mechanically induced stiffening, thermally driven softening, and brittle nature of SiC
}

\author{
Dinesh VARSHNEY ${ }^{a,}$, Swarna SHRIYA ${ }^{a}$, Sanjay JAIN ${ }^{a}$, \\ Meenu VARSHNEY ${ }^{b}$, R. KHENATA ${ }^{c}$ \\ ${ }^{a}$ School of Physics, Vigyan Bhavan, Devi Ahilya University, Khandwa Road Campus, Indore 452001, India \\ ${ }^{b}$ Department of Physics, M. B. Khalsa College, Indore 452002, India \\ ${ }^{c}$ Laboratoire de Physique Quantique et de Modélisation Mathématique (LPQ3M), Département de Technologie, \\ Université de Mascara, Mascara 29000, Algeria
}

Received: April 16, 2015; Revised: July 28, 2015; Accepted: August 12, 2015

(C) The Author(s) 2016. This article is published with open access at Springerlink.com

\begin{abstract}
An effective interionic potential calculation with long range Coulomb, charge transfer interaction, covalency effect, short range overlap repulsion extended, van der Waals interaction, and zero point energy effect is implemented to investigate the pressure dependent structural phase transition (ZnS-type (B3) to NaCl-type (B1) structure), and mechanical, elastic, and thermodynamic properties of silicon carbide $(\mathrm{SiC})$. Both charge transfer interaction and covalency effect are important in revealing the pressure induced structural stability, Cauchy discrepancy, anisotropy factor, melting temperature, shear modulus, Young's modulus, and Grüneisen parameter. We also present the results for the temperature dependent behaviors of normalized volume, hardness, heat capacity, and thermal expansion coefficient. $\mathrm{SiC}$ is mechanically stiffened and thermally softened as inferred from pressure (temperature) dependent elastic constant's behavior. The Pugh's ratio $\phi=B_{\mathrm{T}} / G_{\mathrm{H}}$, the Poisson's ratio $v$, and the Cauchy's pressure $C_{12}-C_{44}$ for $\mathrm{SiC}$ ceramic confirm its brittle nature.
\end{abstract}

Keywords: carbide; computational techniques; phase transition; equation of state

\section{Introduction}

The IV-IV silicon carbide ( $\mathrm{SiC})$ possesses tetrahedral $\mathrm{C}$ and $\mathrm{Si}$ atoms with strong bonds in the lattice. The wide variety of its polytypes possesses unique structural and optical properties [1,2]. SiC usually referred as carborundum material is important due to its wide energy band gap, low dielectric constant, low thermal expansion, and excellent and superior properties of strength, thermal conductivity, hardness,

* Corresponding author.

E-mail: vdinesh33@rediffmail.com melting point, elastic modulus, thermal shock resistance, and chemical inertness.

Previous high pressure experimental $[3,4]$ and theoretical [5-15] research of $\mathrm{SiC}$ infers cubic (3C), hexagonal $(6 \mathrm{H})$, and rhombohedral $(15 \mathrm{R})$ structures. Energy-dispersive X-ray diffraction with a diamond anvil cell shows a structural transition from zinc blende (ZB, B3) to rock salt (RS, B1) at $100 \mathrm{GPa}$ with a volume collapse of about $20.3 \%$ [3]. Transition is reversible and zinc blende phase is recovered below 35 $\mathrm{GPa}$ upon decompression. Furthermore, $6 \mathrm{H}$ polytype $\mathrm{SiC}$ is found to be stable up to about $90.0 \mathrm{GPa}$. Shock compression experiment on $6 \mathrm{H} \mathrm{SiC} \mathrm{shows} \mathrm{a} \mathrm{first-order}$ phase transition into a six-fold coordinated rock salt 
structure at about $105 \pm 4 \mathrm{GPa}$ with a volume reduction of $15 \% \pm 3 \%$ [4].

Structural and thermal stability as well as high pressure behavior of $3 \mathrm{C} \mathrm{SiC}$ has been described by $a b$ initio [5-12] and molecular dynamics simulations [13-16]. The ab initio density functional calculation with the local density approximation (LDA) retraces the transition from zinc blende to rock salt at around $60 \mathrm{GPa}$ [4-7]. Following the Perdew-Wang generalized gradient approximation (GGA) for the exchange correlation potential and the Troullier-Martins pseudopotentials, the transition pressure of about $63 \mathrm{GPa}$ is predicted [8-10]. Using the Troullier-Martins pseudopotentials and the LDA, the phase transition pressure of $100 \mathrm{GPa}$ is also documented [11,12]. It is noted that first-principles LDA calculations underestimate the critical pressure for structural phase transition.

The constant pressure molecular dynamics (MD) simulation for $\mathrm{SiC}$ shows the reversible phase transformation from 3C to rock salt [13]. This demonstrates the structural transformation from a four-fold coordinated structure to a six-fold coordinated structure under pressure. The mechanical properties of $\mathrm{SiC}$ from $\mathrm{B} 3$ to $\mathrm{B} 1$ under pressure are investigated by the first-principles plane-wave pseudopotential density functional theory method [14]. The result on the high pressure elastic constant illustrates that the $\mathrm{ZB}$ structure $\mathrm{SiC}$ is unstable when the applied pressure is larger than $126.6 \mathrm{GPa}$, consistent with the earlier experimental data $[3,4]$ and the molecular dynamics simulation results [13].

The molecular dynamics with effective interatomic interaction potential for $\mathrm{SiC}$ incorporating two-body and three-body covalent interactions is also proposed $[15,16]$. The covalent characteristic $\mathrm{SiC}$ is described by the three-body potential using modified Stillinger-Weber form. The molecular dynamics method with the developed interaction potential is employed to investigate the structural, elastic, and dynamical properties of crystalline 3C, amorphous, and liquid states of $\mathrm{SiC}$ for several densities and temperatures $[15,16]$. The quantum mechanical calculations are powerful probes to generate reference data for the experimentalists and to tailor new structures of materials [17]. The analytical model calculations with nonlocal and long range interactions are substantial for dense molecules and materials, soft matter, van der Waals complexes, and biomolecules [18].
The structure of silicon carbide $(\mathrm{SiC})$ is viewed as stacking sequence of silicon carbon pair layers arrayed in the cubic $(\mathrm{C})$, hexagonal $(\mathrm{H})$, or rhombohedral $(\mathrm{R})$ structure. The cubic (3C) structure has the lowest energy, followed by the wurtzite $(2 \mathrm{H})$ and rock salt (RS) structures as noted by the experimental observations $[3,4]$. The cubic phase of $\mathrm{SiC}$ with zinc blende and wurtzite structures with four-fold coordination transforms into six-fold coordinated ionic RS structure at 105 and $95 \mathrm{GPa}$, respectively. The ground state properties of $\mathrm{SiC}$ in the zinc blende (ZB) (P or $3 \mathrm{C}$ type) and wurtzite $(2 \mathrm{H})$ structures are shown to be in good agreement with experiment. The difference in energy per particle between these two structures is only $\Delta E=0.022 \mathrm{eV}$, zinc blende structure being more stable than wurtzite structure $[15,16]$.

As compared to quantum calculations, the lattice model calculations [19-21] are equally productive in yielding qualitative and quantitative results with proper parameterization of input parameters. For $\mathrm{SiC}$, although structural transitions are known to some extent, but to the best of our knowledge, no systematic efforts have been made to explore the high pressure and high temperature dependence of the aggregate elastic constant, anisotropy, melting temperature, Poisson's ratio, elastic wave velocity, Grüneisen parameter, isothermal (adiabatic) compressibility, shear (Young's) modulus, brittle/ductile nature, hardness, Lamé's constant, Kleinman parameter, and thermodynamic properties as Debye temperature, heat capacity, and thermal expansion coefficient in either $\mathrm{ZnS}$ or $\mathrm{NaCl}$ structure.

The quantum mechanical computational methods based on the density functional theory (DFT) provide reference data for the experimentalists and complete existing theoretical and experimental works on solids and large molecules. For large molecules and large homogeneous systems such as simple metals and semiconductors, the DFT with approximate local and semilocal density functional is appropriate. Furthermore, semilocal density approximations and generalized gradient approximations (GGA) are effective for inhomogeneous systems, such as transition metals, ionic crystals, compound metals, surfaces, interfaces, and some chemical systems.

It is suggested that for dense molecules and materials and sparse systems, including soft matter, van der Waals complexes, and biomolecules, nonlocal long range interactions are influential. In DFT, it introduces only atomic position, space group, and 
value of volume around the experimental volume of the system, and executes the codes to obtain the total energy corresponding to this volume. Later on, equation of state is used to obtain the fundamental (equilibrium) structural properties such as lattice constant of the system, the bulk modulus, and its pressure derivatives. A set of pressure-volume $(P-V)$ values for both phases estimates the transition pressure by the common tangent between the two curves.

The modelling of lattice models in $\mathrm{SiC}$ compound is a complicated task, and in many instances, must be guided by experimental evidence of the low degree of freedom in order to obtain a correct minimal model, which will capture the observed effect and make useful predictions. In comparison to the other quantum mechanical models, phenomenological lattice models are successful as they are predictive and perform the computaion within less frame of time as well as without sophisticated computers and programming tools but with proper parameterisation of the input parameters. We do not claim that the proposed model is the most appropriate and it still needs modifications to have consistency with several properties of $\mathrm{SiC}$ ceramic.

In Section 2, we provide the details of pairwise potential between a pair of ions ( $\mathrm{Si}$ and $\mathrm{C}$ ), and Section 3 discerns the results and the comparison with the experimental and other theoretical studies. We end up with conclusions in Section 4.

\section{Details of calculations}

The understanding of pressure dependent structural properties such as first-order structural phase transition and associated volume collapse, elastic properties such as ductility, mechanical stiffening, thermal softening, anisotropy in elastic constants, shear (Young's) modulus, hardness, Lamé's constant, Kleinman's parameter, shear and longitudinal elastic wave velocity, as well as thermodynamics properties such as Debye temperature, melting temperature, heat capacity, and thermal expansion coefficient of $3 \mathrm{C} \mathrm{SiC}$, needs the formulation of an effective interatomic potential. The idea we have in mind follows: the change in force constants is small, the short range interactions are effective up to the second neighbor ions, and the atoms are held together with harmonic elastic forces without any internal strains within the crystal. Usually, the applications of pressure cause an increase in the overlap of adjacent ions in a crystal, and hence charge transfer takes place between the overlapping electron shells. The transferred charges interact with all others of the lattice via Coulomb's law and give rise to charge transfer interactions under pressure. We have also incorporated zero point energy effect, although it has a small effect in Gibbs free energy.

An isolated phase is stable only when its free energy is minimized for the specified thermodynamic conditions. As the temperature or pressure or any other variable acting on the systems is altered, the free energy changes smoothly and continuously. A phase transition is said to occur when the changes in structural details of the phase are caused by such variations of free energy. The $\mathrm{SiC}$ ceramic transforms from its initial B3 to B1 structure under pressure. The stability of a particular structure is decided by the minima of Gibbs free energy $G=U+P V-T S$, where $U$ is the internal energy which at $0 \mathrm{~K}$ corresponds to the cohesive energy, and $S$ is the vibrational entropy at absolute temperature $T$, pressure $P$, and volume $V$.

We must mention that the calculations presented here assume zero temperature, i.e., the frozen ionic degree of freedom. Although the experimental results are obtained at ambient temperature inferring a certain small temperature dependence of the transition pressure in the range of low temperatures on polycrystalline or bulk samples. It is worth considering the lattice calculation result as a representative of the results that would be obtained under the actual experimental conditions. At zero temperature, the thermodynamically stable phase at a given pressure $P$ is the one with the lowest entropy, and the thermodynamical potential is the Helmholtz free energy $H$.

The Gibbs free energies for $\mathrm{ZnS}$ (B3) phase and $\mathrm{NaCl}$ (B1) phase given by Born equation $G_{\mathrm{B} 3}(r)=$ $U_{\mathrm{B} 3}(r)+3.08 P^{3} \quad$ and $G_{\mathrm{B} 1}\left(r^{\prime}\right)=U_{\mathrm{B} 1}\left(r^{\prime}\right)+2 P r^{\prime 3}$ respectively become equal at the phase transition pressure $P$ and zero temperature, i.e., $G_{\mathrm{B} 1}=G_{\mathrm{B} 3}$ [22]. Here, $V_{\mathrm{B} 3}\left(=3.08 r^{3}\right)$ and $V_{\mathrm{B} 1}\left(=2 r^{\prime 3}\right)$ as the unit cell volumes are the nearest neighbor distances for B3 $(\mathrm{ZnS})$ phase and $\mathrm{B} 1(\mathrm{NaCl})$ phase respectively; the abbreviation $U_{\mathrm{B} 3}(r)$ stands for the $\mathrm{ZnS}$ (B3) phase and $U_{\mathrm{B} 1}\left(r^{\prime}\right)$ for the rock salt (B1) phase, and their relevant expressions are

$$
\begin{aligned}
U_{\mathrm{B} 3}= & \left(-\alpha_{\mathrm{M}} Z e^{2} / r\right)[Z+2 n f(r)]-C r^{-6}-D r^{-8} \\
& +n b \beta_{i j} \exp \left[\left(r_{i}+r_{j}-r_{i j}\right) / \rho\right]
\end{aligned}
$$




$$
\begin{aligned}
& +\left(n^{\prime} b / 2\right)\left\{\beta_{i i} \exp \left[\left(2 r_{i}-k r_{i j}\right) / \rho\right]\right. \\
& \left.+\beta_{j j} \exp \left[\left(2 r_{j}-k r_{i j}\right) / \rho\right]\right\}+\left\{\hbar\left\langle\omega^{2}\right\rangle^{1 / 2} / 2\right\} \\
U_{\mathrm{B} 1}= & \left(-\alpha_{\mathrm{M}}^{\prime} Z e^{2} / r^{\prime}\right)\left[Z+2 m f\left(r^{\prime}\right)\right]-C r^{\prime-6}-D r^{\prime-8} \\
& +m b \beta_{i j} \exp \left[\left(r_{i}+r_{j}-r_{i j}^{\prime}\right) / \rho\right] \\
& +\left(m^{\prime} b / 2\right)\left\{\beta_{i i} \exp \left[\left(2 r_{i}-k^{\prime} r_{i i}^{\prime}\right) / \rho\right]\right. \\
& \left.+\beta_{j j} \exp \left[\left(2 r_{j}-k^{\prime} r_{j j}^{\prime}\right) / \rho\right]\right\}+\left\{\hbar\left\langle\omega^{2}\right\rangle^{1 / 2} / 2\right\}
\end{aligned}
$$

It is known that under compression, the outer most electronic shells of the adjacent ions/atoms overlap. The first term in potential energy is the long rang (LR) Coulomb attraction. The overlapping leads to charge transfer and is the second term in Eqs. (1) and (2). As the ionic charge for each atom cannot be determined uniquely, the calculation of the Madelung energy is modified by incorporating the covalency effect [15-17]. We thus need to incorporate the effective charge arisen due to the polarization of a spherical shaped dielectric in displacing the constituent positive ions $[23,24]$.

The IV-IV SiC semiconducting compound contains covalent bonds, so some electrons are distributed over the region between neighboring atoms. In such situation, energies due to dipole-dipole and dipolequadrupole interactions are of vital importance. The third and fourth terms are the short range (SR) van der Waals attraction. $C$ and $D$ are the overall van der Waals coefficients successfully evaluated from the variational approach [25]. The van der Waals coefficients due to dipole-dipole and dipole-quadrupole interactions are calculated from the Slater and Kirkwood variational approach and the details are given elsewhere [19].

The fifth and sixth terms are short range (SR) repulsive energies due to the overlap repulsion between $i j$, $i i$, and $j j$ ions. $\alpha_{\mathrm{M}}\left(\alpha_{\mathrm{M}}^{\prime}\right)$ is the Madelung constant for B3 (B1) phase. $\beta_{i j}$ is the Pauling coefficient defined as $\beta_{i j}=1+Z_{i} / n_{i}+Z_{j} / n_{j}$ with $Z_{i}\left(Z_{j}\right)$ and $n_{i}\left(n_{j}\right)$ as the valence and the number of electrons in the outermost orbit respectively. The symbols $n(=4)$ and $n^{\prime}(=6)$ are the numbers of the nearest unlike and like neighbors, respectively, for B3 $(\mathrm{ZnS})$, and $m(=6)$ and $m^{\prime}(=6)$ for $\mathrm{B} 1(\mathrm{NaCl})$ structure. $Z e$ is the ionic charge, $k\left(k^{\prime}\right)$ is the structure factor for B3 (B1) structure, and $b(\rho)$ is the hardness (range) parameter. The nearest neighbor ion separation for B3 (B1) structure is $r\left(r^{\prime}\right)$.

The last term is the lowest possible energy of the system and is due to the zero point energy. Here, $\left\langle\omega^{2}\right\rangle^{1 / 2}\left(=k_{\mathrm{B}} \theta_{\mathrm{D}} / \hbar\right)$ is the mean square frequency related to the Debye temperature $\theta_{\mathrm{D}}$. The Debye temperature can be known either from heat capacity measurements or from the bulk modulus value using $\theta_{\mathrm{D}}=\left(\hbar / k_{\mathrm{B}}\right) \sqrt{5 r_{0} B_{\mathrm{T}} / \mu}$. Herein, $r_{0}, B_{\mathrm{T}}$, and $\mu$ are the equilibrium distance, bulk modulus, and reduced mass of the compound, respectively. Henceforth, model potential for ground state incorporates the attractive, repulsive, and zero point energy. Furthermore, the second term in Eqs. (1) and (2) is an algebraic sum of central force part of the charge transfer force parameter, and the force parameter arisen due to covalent nature, i.e., $f(r)=f_{\text {cti }}+f_{\text {cov }}$. The charge transfer force parameter $f_{\text {cti }}$ is expressed as $f_{\text {cti }}=f_{0} \exp (-r / \rho) \quad[20,26,27]$. Here, $r_{i}\left(r_{j}\right)$ is the ionic radius of ion $i(j)$.

Keep in mind that IV-IV SiC semiconducting compound is covalent in bonding, and attractive force due to covalency is important that modifes the effective charge. The polarizing effects originate from changes in covalency due to $\mathrm{Si}-\mathrm{Si}, \mathrm{Si}-\mathrm{C}$, and $\mathrm{C}-\mathrm{C}$ interacting electric fields. The covalency term is expressed as $f_{\text {cov }}(r)=4 e^{2} V_{\text {spo }}^{2}\left(r_{0} E_{\mathrm{g}}^{3}\right)^{-1} \quad$ [19]. Herein, $V_{\text {spo }}$ is the transfer matrix element between the outermost $\mathrm{p}$ orbital and the lowest excited s state, $E_{\mathrm{g}}$ is the transfer energy of electron from $\mathrm{p}$ to $\mathrm{s}$ orbital. The effective charge $e_{\mathrm{S}}^{*}$ of the host crystal is related to the number of electrons transferred to the unoccupied orbitals from its surrounding nearest neighbour, and $n_{\mathrm{c}}=1-e_{\mathrm{S}}^{*} / e$. Henceforth, for overlap distortion effect, $e_{\mathrm{S}}^{*} \neq e$. Furthermore, $n_{\mathrm{c}} / 12 \approx$ $V_{\mathrm{sp} \sigma}^{2} / E_{\mathrm{g}}^{2}$, and the transfer matrix element $V_{\mathrm{sp} \sigma}$ and the transfer energy $E_{\mathrm{g}}$ are related to effective charge $e_{\mathrm{S}}^{*}$ following $V_{\mathrm{sp} \sigma}^{2}\left(E_{\mathrm{g}}^{2}\right)^{-1}=\left(1-e_{\mathrm{S}}^{*}\right) / 12$. The transfer energy $E_{\mathrm{g}}$ is $E_{\mathrm{g}}=E-I+(2 \alpha-1) e^{2} / r$. Here, $E$ is the electron affinity for non metal atom and $I$ is the ionisation potential of constituent metal atom.

The static dielectric constant $\varepsilon_{0}$ and the high frequency dielectric constant $\varepsilon_{\infty}$ are intimately related to Szigeti effective charge $e_{\mathrm{S}}^{*}\left(=Z e^{*}\right)$ [19] as follows: $\quad e_{\mathrm{S}}^{*_{2}}=9 \mu \omega_{\mathrm{TO}}^{2}\left(\varepsilon_{0}-\varepsilon_{\infty}\right)\left[4 \pi N_{k}\left(\varepsilon_{\infty}+2\right)^{2}\right]^{-1}$ and $e_{\mathrm{S}}^{* 2} / e^{2}=\left[9 V \mu \omega_{\mathrm{TO}}^{2}\left(\varepsilon_{0}-\varepsilon_{\infty}\right)\right]\left[4 \pi e^{2}\left(\varepsilon_{\infty}+2\right)^{2}\right]^{-1}$. Here, $\mu$ is the reduced mass, $N_{k}$ is the number of atoms per unit cell volume, i.e., $N_{k}=1 / V, \omega_{\mathrm{TO}}$ is the long wavelength transverse optical phonon frequency. Thus, for $\mathrm{SiC}, e_{\mathrm{S}}^{*}$ deviates from $e$ and is attributed to covalent nature of 
$\mathrm{Si}-\mathrm{Si}, \mathrm{Si}-\mathrm{C}$, and $\mathrm{C}-\mathrm{C}$ bonds. In $\mathrm{SiC}$, "d" like state of Si hybridizes with "p" like state of C.

We need to study the higher order elastic constants, their pressure derivatives, and anisotropy, and their relevant expressions are reported elsewhere [19]. The effective interatomic potential as discussed above for B3 phase contains four material parameters, namely, modified ionic charge, hardness, range, and charge transfer and covalency parameter $\left(Z_{\mathrm{m}}, b, \rho, f(r)\right)$. We can then obtain these values from the equilibrium conditions for $\mathrm{SiC}$ [28].

\section{Results and discussion}

Pressure and temperature as external variables witness new crystal phases appearing in materials and the relative stability of two crystal structures required, and careful computation for stable phase in IV-IV SiC semiconductor is thus challenging. The high pressure experiment results in huge pressure that causes a reduction of the material volume, and the temperature variation will normally produce much smaller changes in the relative stability of different phases.

The phase transition pressure is determined by calculating the Gibbs free energy $G=U+P V-T S$ for the two phases. The Gibbs free energy is the enthalpy $H=U+P V$ at $T=0 \mathrm{~K}$. It is thus physically meaningful to concern with the Gibbs free energy at zero temperature, which is the enthalpy $H$. At $T=0 \mathrm{~K}$, the thermodynamically stable phase at pressure $P$ is the one with the lowest enthalpy, and the zero temperature theory results in consistent agreement with experiment. However, the effects of finite temperature may be significant. The values of thermodynamical potential $G$ or $H$ have been computed using the values of the four material dependent parameters, namely, modified ionic charge, hardness, range, and charge transfer parameter $\left(Z_{\mathrm{m}}, b, \rho, f(r)\right)$ [28].

To estimate the $3 \mathrm{C} \mathrm{SiC}$ ceramic material parameters, we begin by deducing the van der Waals coefficients $C$ and $D$ involved in Eqs. (1) and (2) from the SlaterKirkwood variational method [25]. The estimated crystal parameters are as follows: van der Waals coefficients for $\mathrm{SiC} c_{i i}=28.76 \times 10^{-60} \mathrm{erg} \cdot \mathrm{cm}^{6}, c_{i j}=$ $0.71 \times 10^{-60} \mathrm{erg} \cdot \mathrm{cm}^{6}, \quad c_{j j}=0.047 \times 10^{-60} \mathrm{erg} \cdot \mathrm{cm}^{6}, \quad C=$ $14.07 \times 10^{-60} \mathrm{erg} \cdot \mathrm{cm}^{6}, d_{i i}=14.284 \times 10^{-76} \mathrm{erg} \cdot \mathrm{cm}^{8}, d_{i j}=$ $0.297 \times 10^{-76} \mathrm{erg} \cdot \mathrm{cm}^{8}, \quad d_{j j}=0.002718 \times 10^{-76} \mathrm{erg} \cdot \mathrm{cm}^{8}$, and $D=3.019 \times 10^{-76} \mathrm{erg} \cdot \mathrm{cm}^{8}$. The van der Waals coefficients are influenced by electronic polarizability and have been directly taken from least-squares fit of experimental refraction data $[29,30]$ using additive rule and a Lorentz factor of $4 \pi / 3$.

For the computation purpose, we have used the experimental information on lattice constant $a$ [31], the bulk modulus $B_{\mathrm{T}}$ [32], ionic Ze, effective charge $e_{\mathrm{S}}^{*}$, Debye temperature $\theta_{\mathrm{D}}$ [32], and the second-order aggregate elastic constant $C_{12}\left(C_{44}\right)$ [33]. While estimating the effective charge $e_{\mathrm{S}}^{*}$, the values of optical dielectric constant $\varepsilon_{\mathrm{s}}$, high frequency dielectric constant $\varepsilon_{\infty}$, and the long wavelength transverse optical phonon frequency $\omega_{\text {Tо }}$ are taken from Ref. [34] to have covalency effect.

To reveal the structural phase transition, we minimize the Gibbs free energies $G_{\mathrm{B} 3}(r)$ and $G_{\mathrm{B} 1}\left(r^{\prime}\right)$ for the equilibrium interatomic spacing $r$ and $r^{\prime}$, respectively. Figure 1 illustrates Gibbs free energies $G_{\mathrm{B} 3}(r)$ and $G_{\mathrm{B} 1}\left(r^{\prime}\right)$ as a function of pressure $P$ for $\mathrm{SiC}$. It is noticed that at zero pressure, the Gibbs free energy in B3 crystal phase is more negative; therefore it is thermodynamically and mechanically stable, while B1 is not. As pressure increases, beyond the phase transition pressure $P_{\mathrm{t}}$, the Gibbs free energy for B1 system becomes more negative than that of $\mathrm{B} 3$ phase, so $\mathrm{B} 1$ will be more stable.

Deduced optimized values for $\mathrm{SiC}$ are: ionic radii $r_{i}=0.42 \AA, r_{j}=1.162 \AA$, hardness $b=7.512 \times 10^{-12} \mathrm{erg}$, range $\rho=3.29 \times 10^{-9} \mathrm{~cm}$, charge transfer parameter $f(r)=5.267 \times 10^{-3}$, equilibrium distance $r_{0}=2.18 \AA$, and equilibrium interatomic spacing in $\mathrm{B} 3$ and $\mathrm{B} 1$ phases $r_{0}(\mathrm{~B} 3)=1.89 \AA, r_{0}(\mathrm{~B} 1)=2.11 \AA$, respectively.

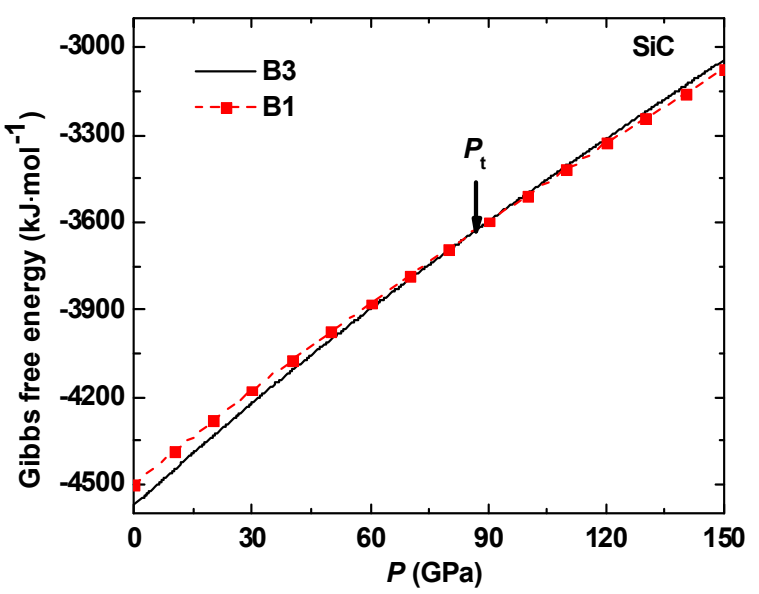

Fig. 1 Variation of Gibbs free energies for B3 and B1 phases with pressure. 
With the above deduced material parameters, we have computed cohesive energy per particle as $6.301 \mathrm{eV}$ for $\mathrm{SiC}$ which is consistent with earlier experimental value of $6.34 \mathrm{eV}$ [35], $a b$ initio density functional calculations (7.41 eV) [36], and molecular dynamics $(6.34 \mathrm{eV})$ for $\mathrm{SiC}[15,16]$. The structural parameters allow to deduce material parameters such as hardness $b$, range $\rho$, and central force as charge transfer force $f(r)$ for the phase stability. We note that $f(r)$ is positive and is attributed to the fact that the charge transfer parameter is computed from the difference of second-order elastic constants $C_{12}$ and $C_{44}$. For $\mathrm{SiC}$, Cauchy energy $C_{12}-C_{44}$ is negative.

In $\mathrm{SiC}$, a crystallographic transition from $\mathrm{B} 3$ to $\mathrm{B} 1$ occurs in certain pressure range. The phase transition pressure $P_{\mathrm{t}}$ thus obtained is listed in Table 1 and compared with available experimental data $[3,4]$ and theoretical results [5-16]. The transition pressure for $\mathrm{SiC}$ is consistent with the experimental and other reported values. The consistency between the experimentally available data and lattice model calculation is attributed to proper formulation of effective interatomic interaction potential, which considers the various interactions explicitly such as the central force as charge transfer interaction, covalency effect, and zero point energy effect, as well as use of material parameters from the experimental data. We must mention that the known computational methods have their own limitations related to the chosen material basic parameter, basic sets, as well as the accuracy and precision used apart from the approximations laid in the methods. Thus, there is a variation in the calculated parameters by each computational method.

As a next step, we have estimated the values of relative volume associated with various compressions following Murnaghan equation of state [37]. To estimate the value of pressure dependent radius for both structures, the curve of volume collapse with pressure to depict the phase diagram is illustrated in Fig. 2 for SiC. The magnitude of the discontinuity in volume at the transition pressure is obtained from the phase diagram and illustrated in Table 1 and is also compared with various experimental $[3,4]$ and other theoretical works [5-14]. It is noticed from Fig. 2 that the present model has predicted correctly the relative stability of competitive crystal structures, as the values of $\Delta G$ are positive. Thus, $\mathrm{SiC}$ with $\mathrm{ZnS}$-type structure

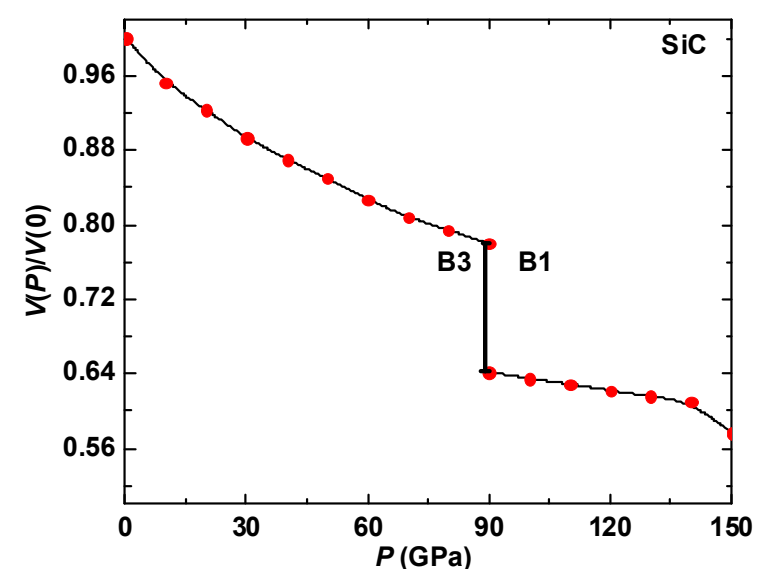

Fig. 2 Equation of state of $\mathrm{SiC}$.

is the stable ground state and the NaCl-type structure is not stable at zero pressure. A phase transition from $\mathrm{NaCl}$ (B1)-type phase (high pressure) to $\mathrm{ZnS}$ (B3)-type phase (ground state) would occur after the pressure quenches to ambient condition.

The calculated aggregate elastic constants at zero pressure are listed in Table 1. The second-order aggregate elastic constants $C_{i j}$ under hydrostatic pressure are obtained with respect to finite strain using the stress-strain coefficients and proper consideration of long range Coulomb and central force as charge transfer interaction and covalency effect, short range overlap repulsion extended up to the second neighbor ions and van der Waals interaction. Figure 3 illustrates the variation of three independent second-order aggregate elastic constants (SOECs) referred as $C_{11}$, $C_{12}$, and $C_{44}$ with external pressure for $\mathrm{SiC}$ in $\mathrm{B} 3$ and $B 1$ phases. Details of method of calculations are earlier reported [19].

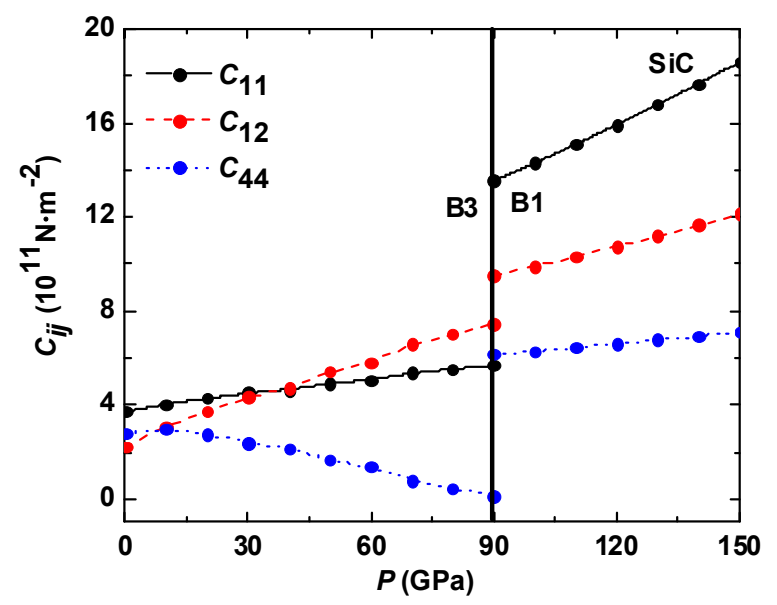

Fig. 3 Variation of aggregate second-order elastic constants with pressure. 
Table 1 Calculated transition pressure $P_{\mathrm{t}}$, volume collapse, aggregate second-order elastic constants $C_{11}, C_{12}$, and $C_{44}$, aggregate bulk modulus $B_{\mathrm{T}}$, pressure derivatives of SOECs $\mathrm{d} B_{\mathrm{T}} / \mathrm{d} P, \mathrm{~d} C_{44} / \mathrm{d} P$, and $\mathrm{d} C s / \mathrm{d} P$, second-order elastic constant anisotropy parameter $\gamma_{1}^{2}$, isotropic shear modulus $G_{\mathrm{H}}$, Voigt's shear modulus $G_{\mathrm{V}}$, Reuss's shear modulus $G_{\mathrm{R}}$, Young's modulus $E$, Poisson's ratio $v$, compressibility $\beta$, Grüneisen parameter $\gamma_{\mathrm{G}}$, Debye temperature $\theta_{\mathrm{D}}$, and melting temperature $T_{\mathrm{m}}$ for $\mathrm{SiC}$ in $\mathrm{B3}$ phase at zero pressure

\begin{tabular}{|c|c|c|c|c|c|c|c|}
\hline Property & Present & Expt. & FP LMTO & $\mathrm{LDF}$ & LDA & MD & DFT \\
\hline Transition pressure $P_{\mathrm{t}}(\mathrm{GPa})$ & 90.0 & $\begin{array}{c}100[3] \\
105 \pm 4[4]\end{array}$ & & & $\begin{array}{l}66 \pm 5[5,6] \\
63[8,9,10]\end{array}$ & $\begin{array}{c}100[13] \\
90[15]\end{array}$ & $\begin{array}{c}66.6[7] \\
100[11] \\
75.4[14]\end{array}$ \\
\hline Volume collapse (\%) & 13.8 & $\begin{array}{c}20.3[3] \\
15 \pm 3[4]\end{array}$ & & & $18.5[5,6]$ & $21[13]$ & $\begin{array}{l}18.5[7] \\
12[11] \\
18[14]\end{array}$ \\
\hline$C_{11}\left(10^{10} \mathrm{~N} \cdot \mathrm{m}^{-2}\right)$ & 37.11 & $\begin{array}{l}39.0[33] \\
36.3[42]\end{array}$ & $\begin{array}{c}42.0[36] \\
35.23[40]\end{array}$ & & $\begin{array}{l}44.9[46] \\
43.6[47]\end{array}$ & $\begin{array}{l}39.0[13] \\
39.0[15]\end{array}$ & $\begin{array}{c}41.51[14] \\
37.1[42]\end{array}$ \\
\hline$C_{12}\left(10^{10} \mathrm{~N} \cdot \mathrm{m}^{-2}\right)$ & 22.34 & $\begin{array}{l}14.2[33] \\
15.4[42]\end{array}$ & $\begin{array}{c}12.6[36] \\
14.04[40]\end{array}$ & & $\begin{array}{l}14.6[46] \\
12.0[47]\end{array}$ & $\begin{array}{c}14.4[13] \\
14.26[15]\end{array}$ & $\begin{array}{c}13.19[14] \\
16.9[42]\end{array}$ \\
\hline$C_{44}\left(10^{10} \mathrm{~N} \cdot \mathrm{m}^{-2}\right)$ & 27.93 & $\begin{array}{l}25.6[33] \\
14.9[42]\end{array}$ & $\begin{array}{c}28.7[36] \\
23.29[40]\end{array}$ & & $\begin{array}{l}25.6[46] \\
25.5[47]\end{array}$ & $\begin{array}{c}17.9[13] \\
19.11[15]\end{array}$ & $\begin{array}{c}26.54[14] \\
17.6[42]\end{array}$ \\
\hline$B_{\mathrm{T}}\left(10^{10} \mathrm{~N} \cdot \mathrm{m}^{-2}\right)$ & 27.30 & $\begin{array}{l}22.7[32] \\
22.4[43] \\
22.5[44]\end{array}$ & $\begin{array}{l}22.3[36] \\
21.1[40]\end{array}$ & $\begin{array}{l}24.9[27] \\
22.5[45]\end{array}$ & $\begin{array}{c}21.2[5,6] \\
22.5[46,47]\end{array}$ & $\begin{array}{c}22.5[13] \\
22.52[15]\end{array}$ & $\begin{array}{c}23.5[11] \\
22.71[14] \\
20.0[39] \\
22.5[42]\end{array}$ \\
\hline$C s\left(10^{10} \mathrm{~N} \cdot \mathrm{m}^{-2}\right)$ & 7.38 & & & & & & \\
\hline $\mathrm{d} B_{\mathrm{T}} / \mathrm{d} P$ & 5.188 & $3.57[32]$ & $3.8[36]$ & $3.2[45]$ & $3.7[5,6]$ & $5.5[15,16]$ & $\begin{array}{c}3.79[14] \\
7.3[39]\end{array}$ \\
\hline $\begin{array}{l}\mathrm{d} C_{44} / \mathrm{d} P \\
\mathrm{~d} C s / \mathrm{d} P\end{array}$ & $\begin{array}{c}5.198 \\
-0.0716\end{array}$ & & & & & & \\
\hline$\gamma_{1}^{2}$ & -0.736 & $2.0[33]$ & $\begin{array}{l}1.95[36] \\
2.20[40]\end{array}$ & & & & \\
\hline$G_{\mathrm{H}}\left(10^{10} \mathrm{~N} \cdot \mathrm{m}^{-2}\right)$ & 16.47 & $19.2[44]$ & $\begin{array}{l}21.9[36] \\
16.9[40]\end{array}$ & & & $12.37[15]$ & $14.1[42]$ \\
\hline$G_{\mathrm{V}}\left(10^{10} \mathrm{~N} \cdot \mathrm{m}^{-2}\right)$ & 19.71 & & $\begin{array}{l}23.1[36] \\
18.2[40]\end{array}$ & & & & $14.6[42]$ \\
\hline$G_{\mathrm{R}}\left(10^{10} \mathrm{~N} \cdot \mathrm{m}^{-2}\right)$ & 13.22 & & $\begin{array}{l}20.8[36] \\
15.7[40]\end{array}$ & & & & $13.6[42]$ \\
\hline$E\left(10^{10} \mathrm{~N} \cdot \mathrm{m}^{-2}\right)$ & 41.22 & $44.8[44]$ & $\begin{array}{c}49.60[36] \\
40.1[40]\end{array}$ & & $\begin{array}{l}56.7[46] \\
55.0[46]\end{array}$ & $31.36[15]$ & $35.2[42]$ \\
\hline$v$ & 0.249 & $\begin{array}{l}0.267[33] \\
0.168[44]\end{array}$ & $\begin{array}{l}0.146[36] \\
0.201[40]\end{array}$ & & & $0.268[15]$ & $\begin{array}{c}1.0[5,6] \\
0.259[42]\end{array}$ \\
\hline$\beta\left(10^{-11} \mathrm{~Pa}^{-1}\right)$ & 0.024 & & & & & & $0.1518[4]$ \\
\hline $\begin{array}{c}\gamma_{\mathrm{G}} \\
\theta_{\mathrm{D}}(\mathrm{K})\end{array}$ & $\begin{array}{c}1.015 \\
611.75\end{array}$ & & & $1.01[45]$ & $1.12[5]$ & & \\
\hline$T_{\mathrm{m}}(\mathrm{K})$ & 2746 & $3100 \pm 40[1]$ & & & & & \\
\hline
\end{tabular}

The second-order elastic constant $C_{11}$ is a measure of resistance to deformation by a stress applied on (100) plane with polarization in the direction $\langle 100\rangle$, and $C_{44}$ refers to the measurement of resistance to deformation with respect to a shearing stress applied across the (100) plane with polarization in the $\langle 010\rangle$ direction. Henceforth, the elastic constant $C_{11}$ represents elasticity in length, and a longitudinal strain produces a change in $C_{11}$. There is no doubt that the elastic constants $C_{12}$ and $C_{44}$ are intimately related to the elasticity in shape, which is a shear constant. However, a transverse strain causes a change in shape without a change in volume. Thus, the second-order aggregate elastic constants $C_{12}$ and $C_{44}$ are less sensitive of pressure as compared to $C_{11}$ in $\mathrm{B} 3$ phase.
As inferred from Fig. 3, $C_{11}$ and $C_{12}$ increase with increase in pressure in both phases. However, $C_{44}$ decreases with the increase of pressure away from zero till the phase transition pressure and then increases in B1 phase. Similar observations have earlier been reported in SiC [14-16]. A crossover of $C_{12}$ and $C_{44}$ in $\mathrm{CaS}$ has also been reported [38]. At phase transition pressure, all the compounds have shown a discontinuity in SOECs, which is in accordance with the first-order character of the phase transition. Thus, we can say that, the developed model potential with emphasis on charge transfer mechanism, covalency, and zero point energy effect consistently explains the high pressure elastic behavior.

According to Born criterion for a lattice to be 
mechanically stable state, the elastic energy density must be a positive definite quadratic function of strain. The principal minors (alternatively the eigen values) of the elastic constant matrix should be all positive at ambient condition. Further, the mechanical stability conditions on the elastic constants in cubic crystals are $B_{\mathrm{T}}=\left(C_{11}+2 C_{12}\right) / 3>0, C_{11}, C_{44}>0$, and $C s=\left(C_{11}-\right.$ $\left.C_{12}\right) / 2>0$ [22]. Here, $C_{i j}$ are the conventional aggregate elastic constants and $B_{\mathrm{T}}$ is the bulk modulus. The quantities $C_{44}$ and $C s$ are the shear moduli of a cubic crystal. Estimated values of bulk modulus $B_{\mathrm{T}}$, shear modulus $C_{44}$, and tetragonal modulus $C s$ well satisfy the above elastic stability criterion for $\mathrm{SiC}$ in $\mathrm{B} 3$ phase and are listed in Table 1.

It should be noted that the second-order elastic constants must depend upon pressure leading to $C_{12}-$ $C_{44} \neq 0$. Henceforth, mechanically stable phases for cubic crystal should satisfy the Born criterion $C_{12}-C_{44}$ $>0$. Referring to equations for $C_{12}$ and $C_{44}$, with emphasis on charge transfer interaction as well as covalency effect, the equilibrium condition leads to $B_{1}+B_{2}=-1.261 Z_{\mathrm{m}}^{2}$. Thus, the second-order elastic constants obtained for optimized values of $r_{i}\left(r_{j}\right)$ leads to Cauchy discrepancy $C_{12}-C_{44} \neq 0$ at zero pressure and zero temperature. The calculated values of pressure derivatives of aggregate second-order elastic constants $\mathrm{d} B_{\mathrm{T}} / \mathrm{d} P, \mathrm{~d} C_{44} / \mathrm{d} P$, and $\mathrm{d} C s / \mathrm{d} P$ are given in Table 1, and are also compared with available experimental $[32,33,42]$ and theoretical [13-16,32, 39-47] studies. The stable phase of the crystal is the one in which the shear elastic constant $C_{44}$ is nonzero and which has the lowest potential energy among the mechanically stable lattices [48].

We note that the calculated values of $C_{12}$ as listed in Table 1 have significant difference with that from experimental values [33,42]. The above difference suggests that the proposed interatomic potetial model needs modification. The hybridised $\mathrm{sp}^{3}$ tetrahedral bonding structure of $\mathrm{SiC}$ in the potential is one of the possibility and can be made successfully sophisticated computational techniques. The present model calculation has a limitation and is the root cause of the above difference in calculated elastic properties when compared with experimental data.

We have studied the elasticity by means of model which assumes that the interatomic forces have a certain shape and directionality. The resulting forces (long range and short range) are only in the direction of the nearest neighbors (central force model). Usually, the Cauchy discrepancy $\Delta_{1}^{2}=C_{12}-C_{44}-2 P$ is a measure of the contribution from the non-central many-body force. However, for pure central interatomic potentials, the Cauchy relation $C_{12}=C_{44}+$ $2 P$ should be satisfied. At zero pressure, the Cauchy discrepancy for $\mathrm{SiC}$ is about $-5.588 \times 10^{10} \mathrm{~N} \cdot \mathrm{m}^{-2}$ which further enhances on increasing the pressure in both phases as evident from Fig. 4. This might be due to the fact that for $\mathrm{SiC}$, non-central charge transfer interaction becomes significant at higher pressures. The significant deviation of $\Delta_{1}^{2}$ at different pressures is the strength of non-central many-body forces as charge transfer interaction and covalency effect as we dealt with.

The anisotropy in second-order elastic constants is of geophysical interest. It is known that anisotropic parameter $\gamma$ is a unity for isotropic elasticity but still the cubic crystal which is isotropic in structure, has elastic anisotropy other than a unity as a result of a fourth rank tensor property of elasticity. The elastic anisotropic parameter $\gamma_{1}^{2}$ of a cubic crystal is defined as $\gamma_{1}^{2}=\left(C_{11}-C_{12}-2 C_{44}\right) / 2 C_{44}$ [49]. Once the secondorder elastic constants are known, it can obtain the elastic anisotropic parameter $\gamma_{1}^{2}$ at various pressures for $\mathrm{SiC}$. Figure 5 illustrates the pressure dependence of the elastic anisotropic parameter $\gamma_{1}^{2}$. It is clear from the plot that below transition pressure as well as at low pressures, anisotropy parameter is insensitive to applied pressures. However, in the vicinity of transition pressure, jump has been noted. Also, for B1 phase, the anisotropy is constant under pressure. We have plotted the elastic anisotropy for both phases. The values of anisotropic parameter $\gamma_{1}^{2}$ for this compound is given in Table 1 at zero temperature and pressure and

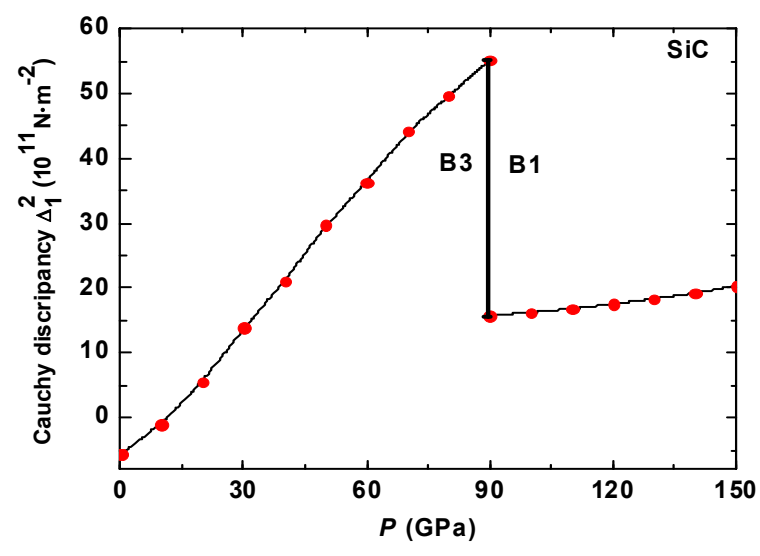

Fig. 4 Variation of Cauchy discrepancy in second-order elastic constant with pressure. 


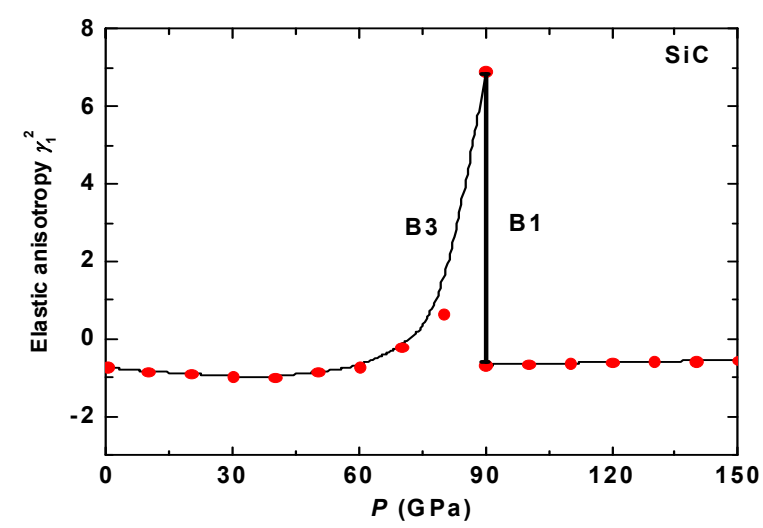

Fig. 5 Variation of aggregate second-order elastic anisotropy $\gamma_{1}^{2}$ with pressure.

compared with the available experimental and theoretical results $[33,36,40,41]$.

Melting ranges are very important during the fabrication and application as the success of the melting and casting operations depends on the correct selection of temperature. Once solidified and primary processed (rolling or forging), the melting temperature has little significance to designers, engineers, and users. Although melting temperature does influence elevated temperature properties such as creep strength, this is only of interest to researchers. The pressure dependence of the melting temperature $T_{\mathrm{m}}=553 \mathrm{~K}+$ $5.91 C_{11} \mathrm{~K} / \mathrm{GPa}$ for $\mathrm{SiC}$ in $\mathrm{B} 3$ and $\mathrm{B} 1$ phases is discerned in Fig. 6. It is noticed that $T_{\mathrm{m}}$ enhances with increased pressure, or in other words, the resistance to deformation by stress increases. An increase in $T_{\mathrm{m}}$ infers the hardening or stiffening of the lattice. $\mathrm{SiC}$ is the only compound in the siliconcarbon binary system. It is noticed that at zero pressure, the melting temperature of $\mathrm{SiC}$ is about $3100 \pm 40 \mathrm{~K}$ [1]. Higher melting temperature symbolizes higher shear

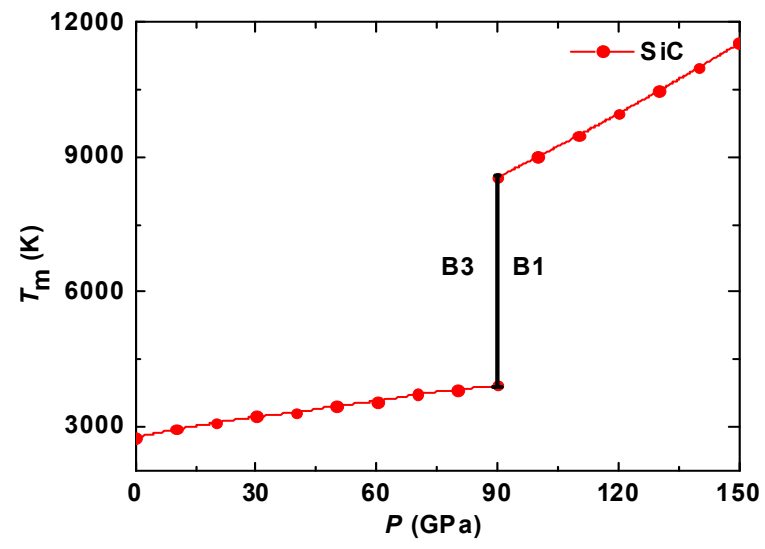

Fig. 6 Variation of melting temperature $T_{\mathrm{m}}$ with pressure. modulus $G$ and Young's modulus $E$. The data on its melting under high pressure are very limited and extremely contradictory, which do not allow to make any conclusions about melting behavior (congruent or incongruent) and the slope of the melting curve of SiC.

The temperature dependence of the third-order elastic constant is noteworthy in revealing the microstructure. The pressure dependence of higher order elastic constants is used to determine the generalized Grüneisen parameter and the elastic waves propagating in different directions. The details of evaluating $C_{i j k}$ are given elsewhere [19]. We thus find that aggregate elastic constants $C_{111}, C_{112}, C_{166}, C_{144}$, and $C_{456}$ are negative and only $C_{123}$ at zero pressure is positive for $\mathrm{SiC}$ ceramic compound in $\mathrm{B} 3$ phase. To the best of our knowledge, no experimental or theoretical data for the third-order elastic constants of $\mathrm{SiC}$ are available. Therefore, the present results are a prediction study. Figure 7 shows the variation of aggregate third-order elastic constants (TOECs) with pressure for $\mathrm{SiC}$ in $\mathrm{B} 3$ and $\mathrm{B} 1$ phases.

It is noticed that (a) the values of $C_{144}$ and $C_{166}$ increase with enhancing pressure while $C_{456}, C_{112}, C_{111}$, and $C_{123}$ show a decreasing trend for $\mathrm{SiC}$; (b) $C_{144}$ is remarkably larger as compared to other TOECs; and (c) values of all aggregate elastic constants $C_{i j k}$ are influenced by pressure dependence. These observations imply that the aggregate elastic constants $C_{i j k}$ are affected by the inclusion of second nearest neighbour interaction, and these are sensitive to the short range interactions. Henceforth, the long range forces as Coulomb, charge transfer interaction, and covalency are effective in $\mathrm{SiC}$ ceramic. Thus, pressure dependence of third-order elastic constants can have a direct means to understand the interatomic forces at

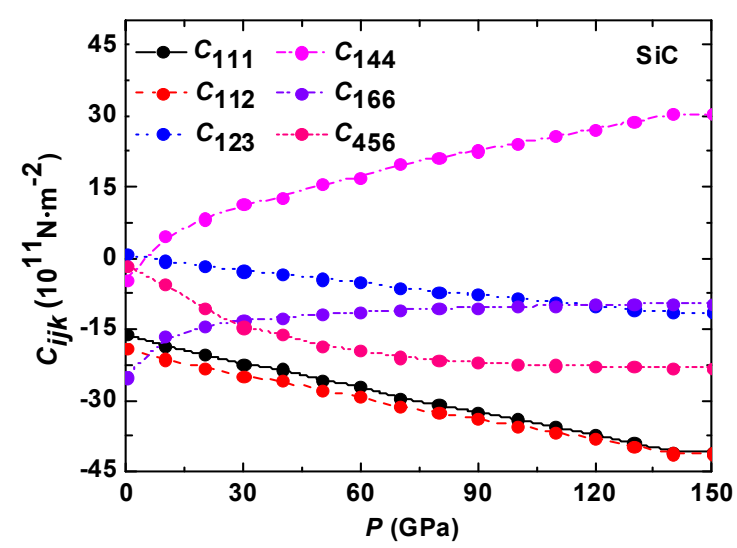

Fig. 7 Variation of aggregate third-order elastic constants with pressure. 
high pressure explicitly, and a balance between long range and short range forces.

In view of equilibrium condition $B_{1}+B_{2}=$ $-1.261 Z_{\mathrm{m}}^{2}$, it is interesting to validate the Cauchy discrepancy $\Delta$ among third-order elastic constants. These are $\Delta_{1}^{3}=C_{112}-C_{166}-2 P, \Delta_{2}^{3}=C_{123}-C_{456}-2 P$, $\Delta_{3}^{3}=C_{144}-C_{456}-2 P$, and $\Delta_{4}^{3}=C_{123}-C_{144}-2 P$. It should be noted that all aggregate elastic constants $C_{i j k}$ in B3 phase contain both long range and short range interactions; hence $\Delta_{i}^{3}$ are the indicators for the contribution from the non-central many-body force. These Cauchy discrepancies are plotted in Fig. 8 as a function of pressure. The significant deviation in $\Delta_{i}^{3}$ is a natural consequence of the non-central many-body force as charge transfer mechanism that we have emphasized. We note that Cauchy discrepancy $\Delta_{i}^{3}$ among all third-order aggregate elastic constants are positive $\left(\Delta_{2}^{3}\right.$ and $\left.\Delta_{3}^{3}\right)$ as well as negative $\left(\Delta_{1}^{3}\right.$ and $\left.\Delta_{4}^{3}\right)$ at zero pressure and become negative at higher pressures in B1 phase indicating the importance of non-central force and anharmonic effect in $\mathrm{SiC}$ ceramic.

For the aggregate third-order elastic constants, there are three anisotropy coefficients and three isotropic coefficients [49]. Figure 9 illustrates the pressure dependence of the elastic anisotropic parameter $\gamma_{i}^{3}$. It is clear from the plot that elastic anisotropies $\left(\gamma_{2}^{3}\right.$ and $\left.\gamma_{3}^{3}\right)$ in B3 phase are less sensitive while $\gamma_{1}^{3}$ shows variation with increase in pressure. We have plotted the elastic anisotropy for both phases. The anisotropy factor $\gamma_{1}^{3}$ shows a decreasing trend with pressure in B1 phase at higher pressures.

Elasticity describes the response of a crystal under external strain as characterized by isotropic shear

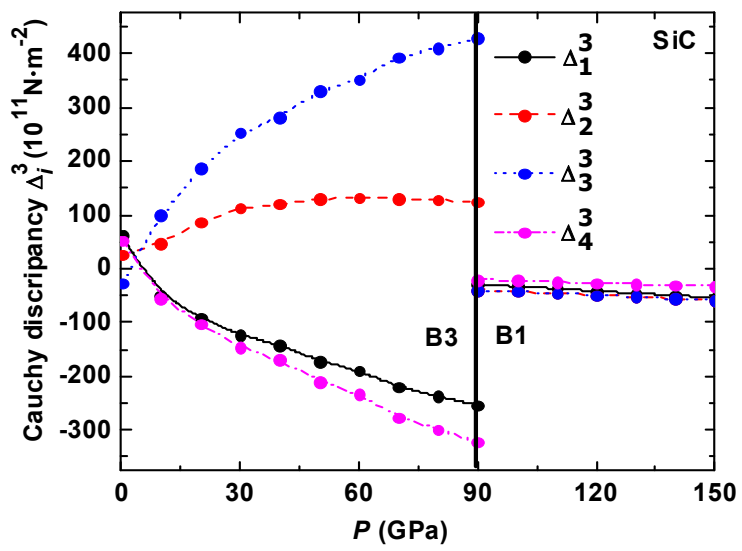

Fig. 8 Variation of Cauchy discrepancy $\Delta_{i}^{3}$ in thirdorder elastic constants with pressure.

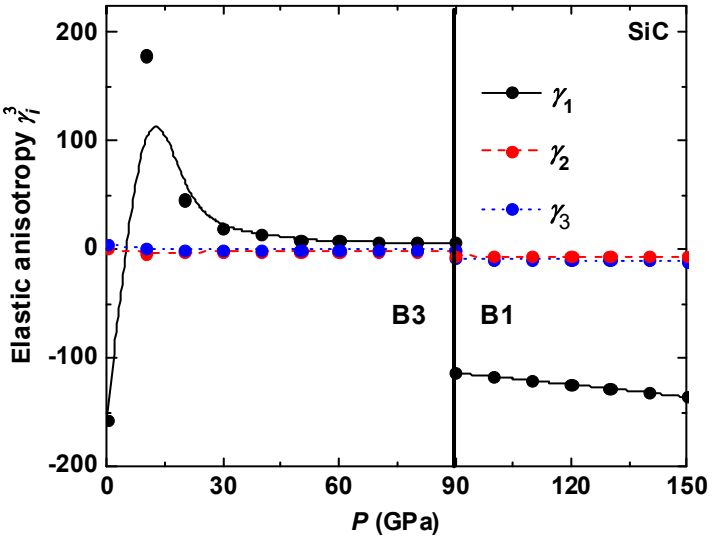

Fig. 9 Variation of third-order elastic anisotropy $\gamma_{i}^{3}$ with pressure.

modulus $G_{\mathrm{H}}$ and Young's modulus $E$. These are often measured for polycrystalline materials when investigating their hardness. On the basis of the Voigt-Reuss-Hill approximation, we have calculated the isotropic shear modulus $G_{\mathrm{H}}$ and Young's modulus $E$ [50-52]. It is known that isotropic shear modulus and bulk modulus are the measure of the hardness for a compound. The bulk modulus is a measure of resistance to volume change by applied pressure, whereas the shear modulus is a measure of resistance to reversible deformation upon shear stress. Therefore, isotropic shear modulus is a better predictor of hardness than the bulk modulus. The calculated isotropic shear modulus $G_{\mathrm{H}}$, Voigt's shear modulus $G_{\mathrm{V}}$, and Reuss's shear modulus $G_{\mathrm{R}}$ are given in Table 1 for $\mathrm{SiC}$ ceramic at zero temperature and pressure and compared with the available theoretical results $[15,16,36,40-42,44]$.

Figure 10 shows that the isotropic shear modulus $G_{\mathrm{H}}$ of $\mathrm{SiC}$ identifies decreasing and increasing trend in B3 phase and then increases with increase in pressure in B1 phase at higher pressures. We note that Voigt's shear modulus $G_{\mathrm{V}}$ is influenced by aggregate elastic constant $C_{44}$; hence $G_{\mathrm{V}}$ decreases as $C_{44}$ decreases with enhanced pressure in $\mathrm{ZnS}$ phase. The Reuss's shear modulus $G_{\mathrm{R}}$ 's pressure dependent behavior is influenced by (a) combination of $C_{11}-C_{12}$ and (b) $C_{44}$. It is noticed that $G_{\mathrm{R}}$ initially decreases and then starts increasing at about $30 \mathrm{GPa}$. Later on, decreasing trend is attributed to steep decrease in $C_{44}$.

From Reuss's shear modulus and bulk modulus, we define the tensile modulus as Young's modulus $E$, and illustrate it for $\mathrm{SiC}$ in Table 1 for $\mathrm{ZnS}$ (B3) phase. The 


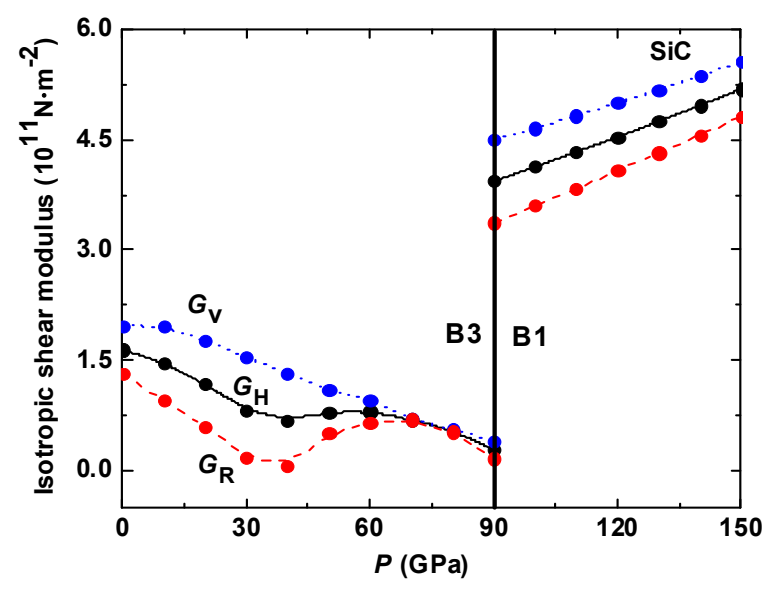

Fig. 10 Variation of isotropic shear moduli $G_{\mathrm{H}}, G_{\mathrm{V}}$, and $G_{\mathrm{R}}$ with pressure.

result is comparable with the available experimental and theoretical results at zero temperature and pressure $[15,16,36,40-42,44,46]$. The material is stiffer if its Young's modulus is high ( $E$ of steel, grapheme, and diamond is about 200, 1000, and $1220 \mathrm{GPa}$, respectively). Compared to graphene and diamond, $\mathrm{SiC}$ compound is less stiff. The pressure dependence of the Young's modulus $E$ of $\mathrm{SiC}$ is illustrated in Fig. 11. A decreasing trend in $\mathrm{B} 3$ phase and an increasing trend in $\mathrm{B} 1$ phase of $E$ are inferred.

Furthermore, the mechanical properties as ductility and brittleness of carbon based compounds are important in technology and can be easily known from second-order elastic constants. We refer to Pugh [53], who suggested an empirical relation relating the plasticity and elastic moduli of materials. Accordingly, the shear modulus $G_{\mathrm{H}}$ of the material refers to the resistance to plastic deformation, and the bulk modulus $B_{\mathrm{T}}$ represents the resistance to fracture. The higher value of ratio $\phi=B_{\mathrm{T}} / G_{\mathrm{H}}>1.75$ reflects the brittle

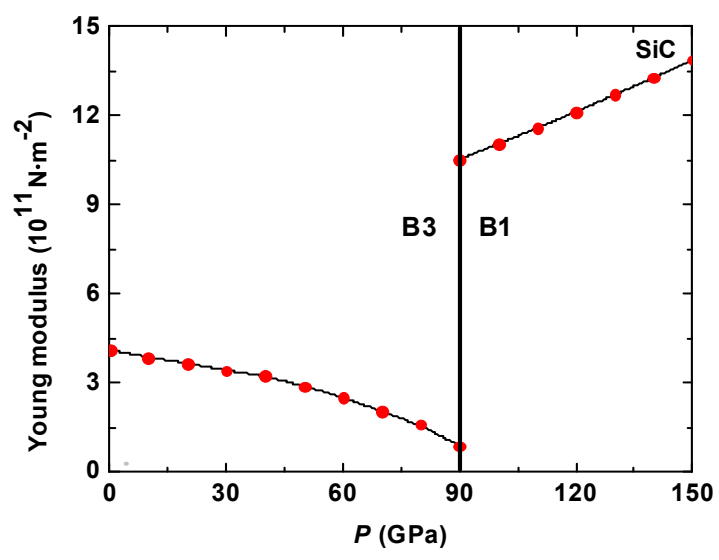

Fig. 11 Variation of Young's modulus $E$ with pressure. nature of the material; otherwise the material is ductile. The critical value that distinguishes the ductile and brittle nature is about 1.75. From Fig. 12, the Pugh's ratio $\phi$ shows that (a) at zero pressure $(\phi=1.656)$ $\mathrm{SiC}$ ceramic is ductile but it becomes brittle with an increase in pressure both in zinc blende (ZB) (B3) and rock salt (RS) (B1) phases. As the value of $\phi=1.656$ at zero pressure is close to the limiting value of 1.75 , it can classify $\mathrm{SiC}$ to be a brittle material. Perovskite materials usually document ductile behavior and the ductility of a material is a measure of the extent to which a material will deform before fracture. It is argued that ductility is also used as a quality control measure to assess the level of impurities and proper processing of material.

Frantsevich et al. [54] further elaborated the ductility and brittleness of materials in terms of Poisson's ratio. According to Frantsevich's rule, the critical value of Poisson's ratio of a material that separates ductile and brittle nature is about 0.33 . For ductile material, the Poisson's ratio is larger than 0.33; otherwise the material behaves in a brittle manner ( $v$ $<1 / 3$ ). Usually, the Poisson's ratio lies in between -1.0 and 0.5 which are the lower and upper bounds respectively. The lower bound is where the material does not change its shape and the upper bound is where the volume remains unchanged. Thus, it is defined the Poisson's ratio $v$ in terms of bulk modulus $B_{\mathrm{T}}$ and the shear modulus $G_{\mathrm{H}}$ as $v=0.5\left(3 B_{\mathrm{T}} / G_{\mathrm{H}}-2\right)$. $\left(3 B_{\mathrm{T}} / G_{\mathrm{H}}+1\right)^{-1} \quad[50-52]$.

From Fig. 12 the Poisson's ratio $v$ shows that (a) at zero pressure $3 \mathrm{C} \mathrm{SiC}$ compound is brittle $(v=0.249)$ in nature and (b) with an increase in pressure, $3 \mathrm{C} \mathrm{SiC}$

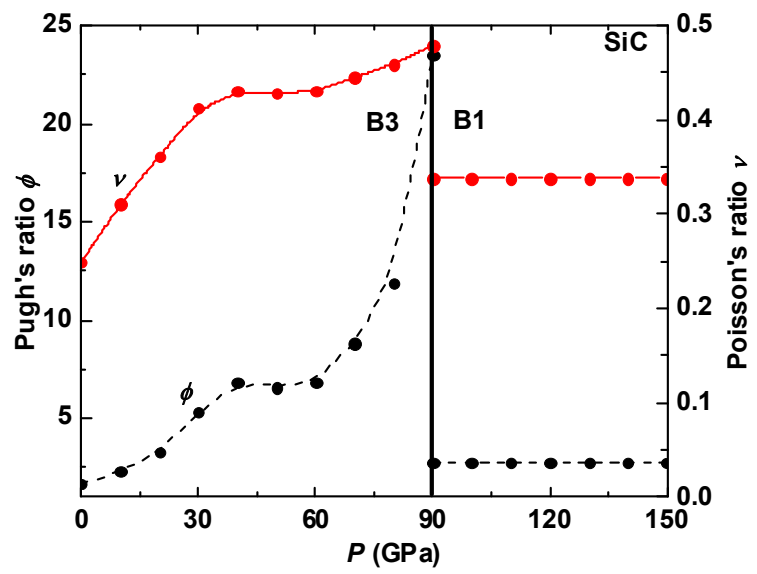

Fig. 12 Variation of Poisson's ratio $v$ and Pugh's ratio $\phi$ with pressure. 
further illustrates the brittle nature in $\mathrm{ZB}$ and $\mathrm{RS}$ phases. Thus, both Pugh's and Frantsevich's rules confirm the brittle nature of $3 \mathrm{C} \mathrm{SiC}$ ceramic. Furthermore, at zero pressure, the value of $v$ is about 0.249 for $\mathrm{SiC}$ and it is in good agreement with available experimental and theoretical results $[5,6,15$, $16,33,36,40-42,44]$. It is noted that the values of Poisson's ratio $v$ for covalent materials are small $(v \approx 0.1)$, whereas for metallic materials $v$ is typically 0.33 .

The brittle behavior of $\mathrm{SiC}$ ceramic can also be checked by Cauchy pressure. Based on the calculated $C_{12}-C_{44}$ for SiC as shown in Table 1 and Table 2, it can be seen that the Cauchy pressures are negative. These results again indicate that $\mathrm{SiC}$ is brittle in nature. Furthermore, Poisson's ratio $v$ is used as a probe to identify the bonding characteristic of the forces other than any other elastic constants. Estimated Poisson's ratio is close to 0.25 , which means that $\mathrm{SiC}$ is with predominantly central interatomic forces. The larger Poisson's ratio is, the better the plasticity is henceforth. $\mathrm{SiC}$ is the one which shows a better plasticity.

Table 2 Calculated aggregate second-order elastic constants $C_{11}, C_{12}$, and $C_{44}$, aggregate bulk modulus $B_{\mathrm{T}}$, second-order elastic constant anisotropy parameter $\gamma_{1}^{2}$, isotropic shear modulus $G_{\mathrm{H}}$, Voigt's shear modulus $G_{\mathrm{V}}$, Reuss's shear modulus $G_{\mathrm{R}}$, Young's modulus $E$, Poisson's ratio $v$, first and second Lamé's constants $\lambda$ and $\mu$, longitudinal $v_{1}$, shear $v_{s}$ and average elastic wave velocity $v_{\mathrm{m}}$, Kleinman parameter $\xi$, and thermodynamical properties: Debye temperature $\theta_{\mathrm{D}}$ and melting temperature $T_{\mathrm{m}}$ of $\mathrm{SiC}$ in $\mathrm{B3}$ phase at about $300 \mathrm{~K}$

\begin{tabular}{cc}
\hline Property & Present \\
\hline$C_{11}\left(10^{10} \mathrm{~N} \cdot \mathrm{m}^{-2}\right)$ & $67.65,29.0[68]$ \\
$C_{12}\left(10^{10} \mathrm{~N} \cdot \mathrm{m}^{-2}\right)$ & $8.31,23.5[68]$ \\
$C_{44}\left(10^{10} \mathrm{~N} \cdot \mathrm{m}^{-2}\right)$ & $19.99,55.0[68]$ \\
$B_{\mathrm{T}}\left(10^{10} \mathrm{~N} \cdot \mathrm{m}^{-2}\right)$ & $28.1,25.0[68]$ \\
$C s\left(10^{10} \mathrm{~N} \cdot \mathrm{m}^{-2}\right)$ & $29.7,2.75[68]$ \\
$\gamma_{1}^{2}$ & 0.484 \\
$G_{\mathrm{H}}\left(10^{10} \mathrm{~N} \cdot \mathrm{m}^{-2}\right)$ & 23.43 \\
$G_{\mathrm{V}}\left(10^{10} \mathrm{~N} \cdot \mathrm{m}^{-2}\right)$ & 23.86 \\
$G_{\mathrm{R}}\left(10^{10} \mathrm{~N} \cdot \mathrm{m}^{-2}\right)$ & 22.99 \\
$E\left(10^{10} \mathrm{~N} \cdot \mathrm{m}^{-2}\right)$ & $54.9,43.7[69]$ \\
$v$ & $0.174,0.167[69]$ \\
$\lambda\left(10^{10} \mathrm{~N} \cdot \mathrm{m}^{-2}\right)$ & 12.47 \\
$\mu\left(10^{10} \mathrm{~N} \cdot \mathrm{m}^{-2}\right)$ & 23.43 \\
$v_{1}(\mathrm{~m} / \mathrm{s})$ & $12440,12182[69]$ \\
$v_{\mathrm{s}}(\mathrm{m} / \mathrm{s})$ & $7819,7701[69]$ \\
$v_{\mathrm{m}}(\mathrm{m} / \mathrm{s})$ & 1824 \\
$\xi$ & 0.274 \\
$\theta_{\mathrm{D}}(\mathrm{K})$ & 708.60 \\
$T_{\mathrm{m}}(\mathrm{K})$ & 4551 \\
\hline
\end{tabular}

We further add that atomistic aspects of ductile responses of extremely hard and brittle $\mathrm{SiC}$ ceramic during nanometric cutting operations have been studied using molecular dynamics simulation [55]. It is suggested that $\mathrm{SiC}$ can be machined in ductile regime at nanoscale through single-point diamond turning process; the root cause of the ductile response of $\mathrm{SiC}$ has not been understood. It is earlier known that slow feed rate helps to achieve high pressure phase transformations which cause ductile responses [56]. It is suggested that the size scale for the ductile-to-brittle transition occurs in the range associated with nanotechnology applications $(10-100 \mathrm{~nm})$. The ductile regime machining of single crystal $\mathrm{SiC}$ occurs at penetration depth or chip thickness less than $500 \mathrm{~nm}$. The ductile behavior of $\mathrm{SiC}$ is further confirmed by production of smooth surfaces and chips indicative of ductile machining similar to metals.

The response of the elasticity is further investigated through Lamé's constants $(\lambda, \mu)$ which in turn yield the compressional velocity and shear wave velocity. The compressional wave propagates back and forth in a crystal, while shear wave goes up and down. The velocities of the longitudinal and shear waves, designated $v_{1}$ and $v_{\mathrm{s}}$ respectively, are known once Lamé's constants $\mu$ and $\lambda$ are determined.

The Lamé's constants $\lambda=v E /[(1+v)(1-2 v)], \quad \mu=$ $E / 2[(1+v)]$ are derived from Young's modulus and Poisson's ratio. Physically, the first Lamé's constant $\lambda$ is a measure of the compressibility of the material, whereas the second Lamé's constant $\mu$ reflects its shear stiffness [50-52], Usually, the shear modulus $\mu$ is positive. The Lamé's first parameter $\lambda$ can be negative, in principle; however, for most materials it is also positive. The two parameters together constitute a parameterization of the elastic moduli for homogeneous isotropic media. The calculated Lamé's constants are $\lambda=16.29 \times 10^{10} \mathrm{~N} \cdot \mathrm{m}^{-2}, \mu=16.47 \times 10^{10} \mathrm{~N} \cdot \mathrm{m}^{-2}$ for $\mathrm{SiC}$ Systematic trend in values of Lamé's constants $(\lambda, \mu)$ is noticed on increasing pressure for SiC. The pressure dependence of the first and second Lamé's constants $(\lambda$, $\mu$ ) are shown in Fig. 13. It is noticed that (a) both Lamé's constants $(\lambda, \mu)$ are positive, and (b) increasing trend of $\lambda$ is in both phases, while $\mu$ first decreases in B3 phase and then increases in B1 phase.

We can obtain the longitudinal (shear) wave velocity $v_{1}=[(\lambda+2 \mu) / \rho]^{1 / 2}, \quad v_{\mathrm{s}}=(\mu / \rho)^{1 / 2}$, and $v_{\mathrm{m}}=\left[\left(2 / v_{1}^{3}+\right.\right.$ $\left.\left.1 / v_{\mathrm{s}}^{3}\right) / 3\right]^{1 / 3}$ from Lamé's constants $(\lambda, \mu)$. The 


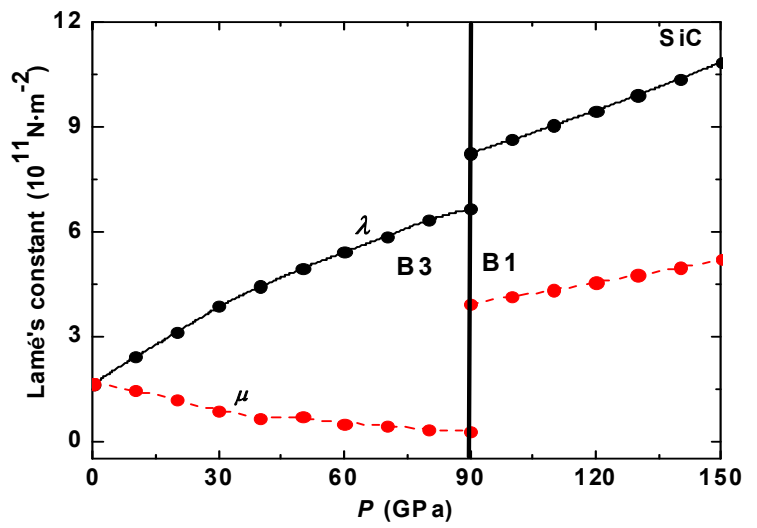

Fig. 13 Variation of Lamé's constants $(\lambda, \mu)$ with pressure.

pressure dependence of the longitudinal (shear) velocity is shown in Fig. 14. It is noticed that $v_{1}$ increases while $v_{\mathrm{s}}$ decreases with increased pressure in B3 phase. Both $v_{1}$ and $v_{\mathrm{s}}$ increase with enhanced pressure in B1 phase. Deduced values of longitudinal $\left(v_{1}=12400 \mathrm{~m} / \mathrm{s}\right)$, shear $\left(v_{\mathrm{s}}=7172 \mathrm{~m} / \mathrm{s}\right)$, and average elastic wave velocity $\left(v_{\mathrm{m}}=1688 \mathrm{~m} / \mathrm{s}\right)$ are propagating in $\mathrm{SiC}$ at zero temperature and pressure.

We may add that the longitudinal and shear wave velocities are also obtained in terms of Reuss's shear modulus $G_{\mathrm{R}}$ and bulk modulus $B_{\mathrm{T}}$ from Navier's equation [57]. We admit that Lamé's constants $(\lambda, \mu)$ are perhaps of substantial interest in materials that are plastic in origin. As Poisson's ratio increases, Lamé's constants will numerically approach the bulk modulus. The Reuss's shear modulus $G_{\mathrm{R}}$ essentially disappears as the viscosity of the fluid approaches zero. The above can also be cross-checked from the relation between bulk modulus and Reuss's shear modulus $B_{\mathrm{T}}=\lambda+$ $2 G_{\mathrm{R}} / 3$.

$G_{\mathrm{R}}$ approaches zero for fluids and hence the

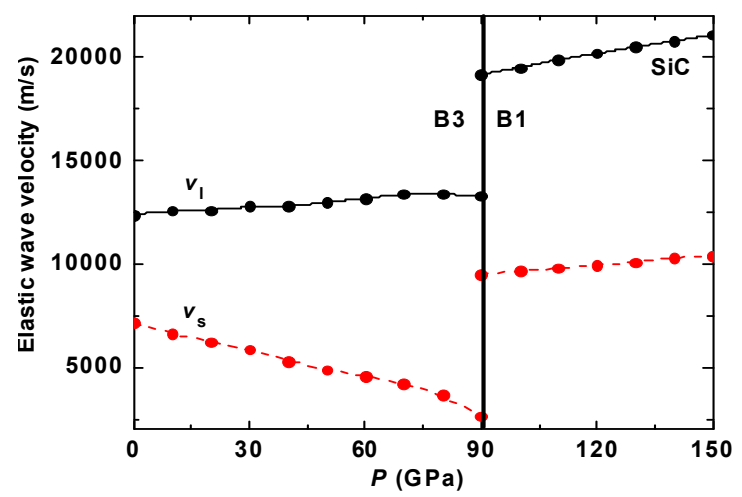

Fig. 14 Pressure dependence of elastic wave velocities $v_{1}$ and $v_{\mathrm{s}}$.
Poisson's ratio is $\sim 0.5$. We thus argue that the studies of Lamé's constants in fluids are of substantial interest as the compressional (shear) velocity $v_{1}\left(v_{\mathrm{s}}\right)$ is proportional to the Lamé's constants or the bulk modulus. Usually, the elastic constants relate the properties of material that undergo stress, deform, and then recover after return to its original shape after stress ceases. The elastic constants are emphasized in solids because they are closely intimated to various fundamental solid state phenomena such as interatomic bonding, equations of state, and phonon spectra. As suggested by Bouhemadou and researchers $[58,59]$, the above is applicable for $\mathrm{SiC}$ as well, and we have attempted to understand the Kleinman parameter $\xi$ which describes the relative positions of the cation and anion sub lattices under volume-conserving strain distortions for which positions are not fixed by symmetry. We use $\xi=C_{11}+8 C_{12} /\left(7 C_{11}+2 C_{12}\right)$ [60].

The Kleinman parameter $\xi=0.709$ is calculated for $\mathrm{SiC}$ at zero temperature and pressure for $\mathrm{ZnS}$ (B3) phase. A low value of $\xi$ implies a large resistance against bond bending or bond-angle distortion and vice versa [61]. Because of unavailability of data, we could not compare them and it can be considered as a prediction of elastic properties. Henceforth, knowledge of elastic constants at variable pressure is substantial for practical applications related to the mechanical properties of a solid: load deflection, thermo elastic stress, internal strain, elastic wave velocities, and fracture toughness.

It is worth to mention that the elastic properties are also linked with thermodynamical properties as heat capacity, thermal expansion, Debye temperature, and Grüneisen parameter. In order to describe the anharmonic properties of a crystal, we have calculated Grüneisen constant $\gamma_{\mathrm{G}}$ and isothermal compressibility $\beta$. Details are given elsewhere [19]. The above thermodynamic parameters are listed in Table 1 . The Grüneisen constant as a function of pressure is plotted in Fig. 15 for $\mathrm{SiC}$ for both phases.

Usually the value of Grüneisen parameter for most of the solids is in between 1.5 and 2.5. The value of $\gamma_{\mathrm{G}}$ for 3C SiC compound is illustrated in Table 1 for $\mathrm{ZnS}$ (B3) phase and it is in good agreement with the available theoretical results $[5,6,45]$. It is evident from the figure that the Grüneisen parameter decreases linearly with increase in pressure. However, there is a 


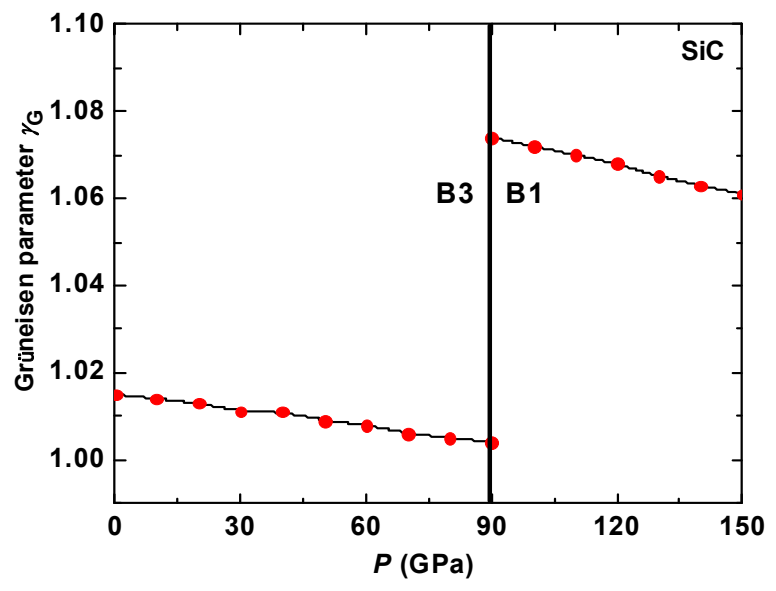

Fig. 15 Grüneisen parameter $\gamma_{G}$ as a function of pressure.

jump in B1 phase and is attributed to the fact that anharmonicity is significant in $\mathrm{SiC}$ ceramic. The Grüneisen parameter jump $\gamma_{\mathrm{G}}(\mathrm{B} 3-\mathrm{B} 1)$ at $P_{\mathrm{T}}$ is about $7 \%$ in $\mathrm{SiC}$. Further investigations are required from knowledge of phonon frequencies as a function of crystal volume $V$.

The variation of isothermal compressibility with pressure is shown in Fig. 16. At zero pressure, the value of $\beta$ is about 0.024 for $\mathrm{SiC}$ and is compared with available theoretical result of 0.1518 [5,6]. It is noticed that in $\mathrm{SiC}$ compound, the lattice is stiffened with increased pressure in B3 phase, gets softened at phase transition pressure, and again becomes stiff at higher pressures, i.e., in B1 phase as reflected from isothermal compressibility $\beta$ behavior. The mechanical stiffened bulk modulus in $\mathrm{SiC}$ is attributed to $\mathrm{Si}-\mathrm{Si}$, $\mathrm{C}-\mathrm{C}$, and $\mathrm{Si}-\mathrm{C}$ bond compression and bond strengthening due to lattice vibration in both B3 and B1 phases. Especially, the compressibility at zero pressure is reduced by about $1 \%$ of that at transition

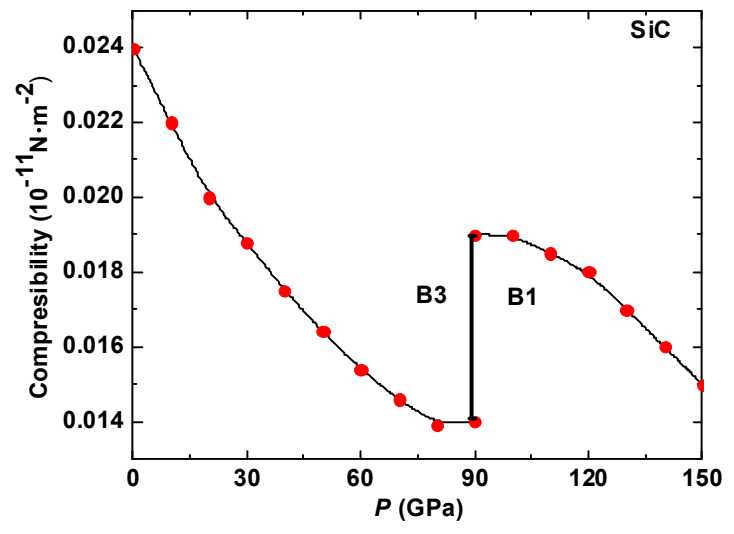

Fig. 16 Variation of aggregate isothermal compressibility with pressure. pressure in $\mathrm{B} 3$ phase in $\mathrm{SiC}$. However, the magnitude of $\beta$ at transition pressure is about $0.3 \%$ of that at higher pressures $(\sim 150 \mathrm{GPa})$. Thus, giant lattice softening is noticed at the boundary of B3-B1 phase transition in $\mathrm{SiC}$.

In a true sense, compressibility is intimately related to the performance of a material, such as elasticity, extensibility, elastic wave velocity, Debye temperature, heat capacity, and thermal conductivity, and is a constant value at ambient atmosphere. Furthermore, compressibility becomes tunable with the applied temperature as well as pressure stimuli. We comment that, the computational details infer that the pressure dependent mechanical induced softening of the lattice at phase transition pressure in $\mathrm{SiC}$ is attributed to enhancement of the cohesive energy as well as increase in the bulk modulus in B3 phase as compared to B1 phase. We have used the effective interatomic potential with emphasis on charge transfer mechanism, covalency effect, and zero point energy effect in $\mathrm{SiC}$ to predict successfully the elastic and anharmonic properties of the ceramic under consideration. Apart from phase transition and pressure dependence of SOECs, we also estimate Debye temperature $\theta_{\mathrm{D}}$ from the present approach.

The elastic constants determine the velocity of elastic waves through the lattice and hence one can relate the Debye temperature $\theta_{\mathrm{D}}$ with the elastic constants, since $\theta_{\mathrm{D}}$ may be estimated from the average sound velocity $v_{\mathrm{m}}$ using $\theta_{\mathrm{D}}=\left(h v_{\mathrm{m}} / k\right)\left(3 n N_{\mathrm{A}} \rho /\right.$ $8 \pi)^{0.33}$ [62-64]. Here, $h$ is Planck's constant, $k$ is Boltzmann's constant, $N_{\mathrm{A}}$ is Avogadro's number, $n$ is the number of atoms in the molecule, $M$ is the molecular weight, $\rho$ is the density, and $v_{\mathrm{m}}$ is average wave velocity. To explain the variation of $\theta_{\mathrm{D}}$ with the pressure, we attempt to analyze our results in the framework of dynamics of lattice with pressure.

The change in the force constants induced by pressure decreases $\theta_{\mathrm{D}}$ in $\mathrm{B} 3$ phase, and after transition pressure it starts increasing which drives the system effectively towards the softening of lattice with increasing pressure. The variation seen in $\theta_{\mathrm{D}}$ in $\mathrm{B} 3$ phase is in accordance with the competitive behavior of pressure dependent aggregate elastic constants $C_{i j}$. The Debye temperature from B3 to B1 phase jumps by $655 \mathrm{~K}$ at $P_{\mathrm{T}}$ in $\mathrm{SiC}$ compound. Usually, the Debye temperature is also a function of temperature and varies from technique to technique as well as depends 
on the sample quality with a standard deviation of about $15 \mathrm{~K}$. The Debye temperature as a function of pressure is plotted in Fig. 17 for $\mathrm{SiC}$ compound. It is noticed from the figure that $\theta_{\mathrm{D}}$ increases with increasing in pressure for $\mathrm{SiC}$ compound and the calculated value is listed in Table 1.

Usually, the elastic moduli describe only reversible response of a material to small strain near equilibrium. The intrinsic strength of a material reflects permanent plastic deformation at large shear strain. Consequently, to further understand the behavior of $\mathrm{SiC}$ is in terms of Vickers hardness $H_{\mathrm{V}}=2\left(\kappa^{2} G\right)^{0.585}-3$. Figure 18 illustrates the theoretical Vickers hardness as a function of pressure. It is clear from the plot that the Vickers hardness $H_{\mathrm{V}}$ decreases in $\mathrm{B} 3$ phase and then increases in B1 phase with increase in pressure, which indicates that $\mathrm{SiC}$ becomes hard under pressure implying its good mechanical properties.

The pressure dependence of Debye temperature is earlier discussed. The variations of heat capacity at

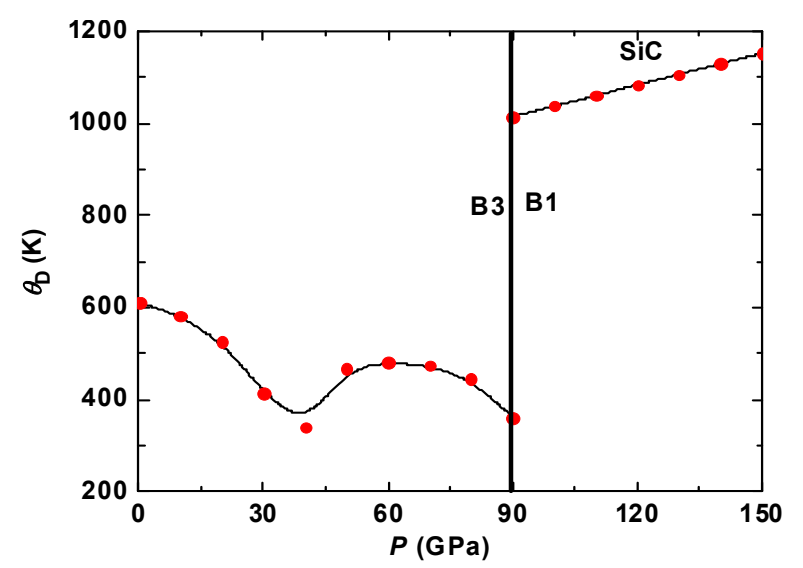

Fig. 17 Debye temperature $\theta_{\mathrm{D}}$ as a function of pressure.

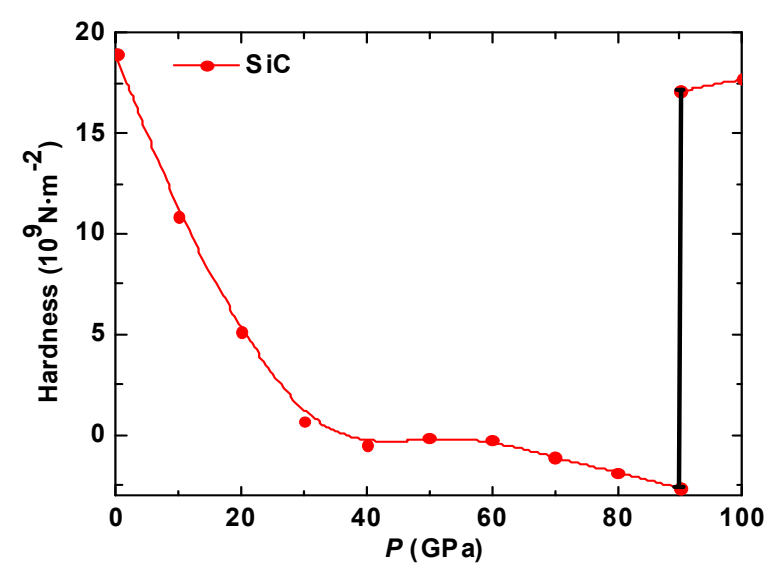

Fig. 18 Hardness $H_{\mathrm{v}}$ as a function of pressure. constant volume, $C_{V}$, with pressure $P$ of $\mathrm{SiC}$ is illustrated in Fig. 19 at $T=600,800,1000$, and $1200 \mathrm{~K}$. We have plotted the normalized heat capacity $\left(C_{V}-C_{V}(0)\right) / C_{V}(0)$, where $C_{V}$ and $C_{V}(0)$ are the heat capacity at any pressure $P$ and at zero pressure respectively. The heat capacity at different temperatures decreases nonlinearly with the applied pressures. It infers that that the frequency vibration of the particles in $\mathrm{SiC}$ compound changes with pressure as well as temperature. For higher temperatures $T \rightarrow \theta_{\mathrm{D}}$, the variation in heat capacity with pressure is weak and at $P_{\mathrm{T}}$, reduced jump in between $\mathrm{B} 3$ and $\mathrm{B} 1$ phases can be seen as compared to low temperatures.

The pressure dependent Grüneisen parameter and Bulk modulus are required apart from heat capacity at constant volume $C_{V}$ to elucidate the thermal expansion coefficient. The thermal expansion coefficient $\alpha_{\text {th.exp }}$ describes any alteration in frequency of the crystal lattice vibration depending on the lattice's expansion or contraction in volume as a result of variation in temperature. We have determined the pressure dependence of $\alpha$ as shown in Fig. 20 for $\mathrm{SiC}$. It can be seen that the thermal expansion coefficient $\alpha_{\text {th.exp }}$ decreases nonlinearly with the pressure for both $\mathrm{ZnS}$ and $\mathrm{NaCl}$ phases. However, the thermal expansion coefficient in higher temperature decreases rapidly with pressure than that in lower temperature. At $P_{\mathrm{T}}$, the thermal expansion coefficient is decreased by $26 \%$ to $22 \%$ in $\mathrm{SiC}$, at temperatures $600,800,1000$, and $1200 \mathrm{~K}$.

While understanding the anharmonic effects on the elastic constants at higher temperatures, we follow the equation of state and the derivatives of energy are

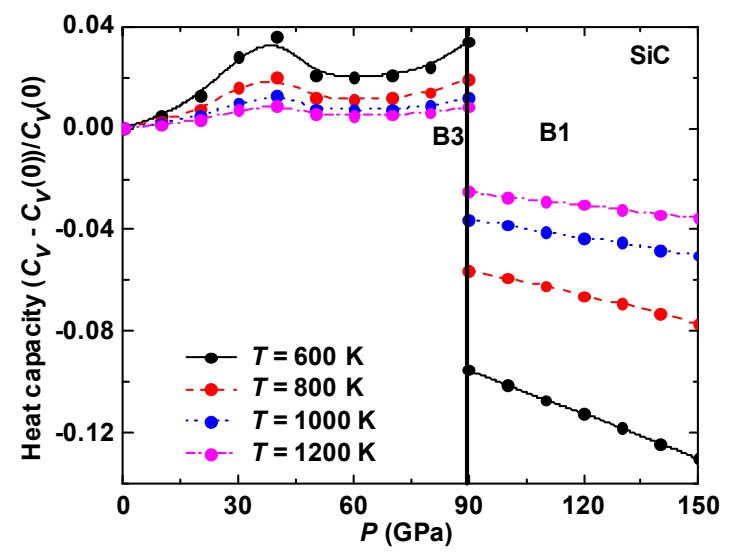

Fig. 19 Variation of heat capacity $C_{V}$ with pressure at different temperatures. 


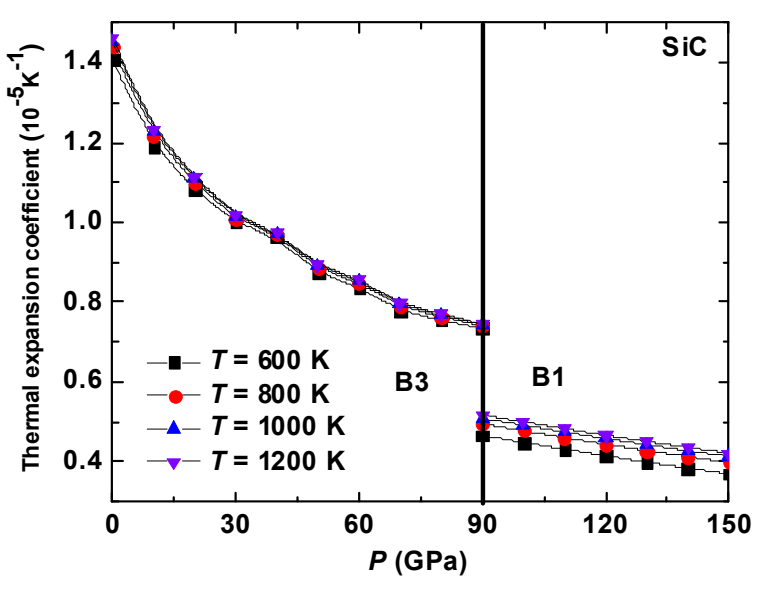

Fig. 20 Variation of the thermal expansion coefficient with pressure at different temperatures.

required to estimate the elastic constants [19]. Figure 21 discerns the variation of $V(T) / V(0)$ as a function of temperature in B3 phase. Here, $V(T)$ symbolizes the volume at various temperatures and $V(0)$ at zero temperature and zero pressure. A steep increase in the ratio $V(T) / V(0)$ with increasing temperature indicates net expansion and $\mathrm{SiC}$ is susceptible to temperature. However, if we refer to Fig. 2, it is noticed that $\mathrm{SiC}$ is compressed at higher pressures. It implies that $\mathrm{SiC}$ is thermally softened and mechanical stiffened. The normalized volume $V(T) / V(0)$ dependence on temperature is not known for $\mathrm{SiC}$, but the present behavior is consistent with available experimental [65] and theoretical [66,67] data on $\mathrm{Li}_{2} \mathrm{O}$.

Figure 22 exhibits the aggregate elastic constants $C_{i j}(T)$ with temperature $T$ for $\mathrm{SiC}$. The values are listed in Table 2 and compared with the available data [68] at room temperature. It can be seen that the aggregate elastic constants $C_{i j}(T)$ decrease

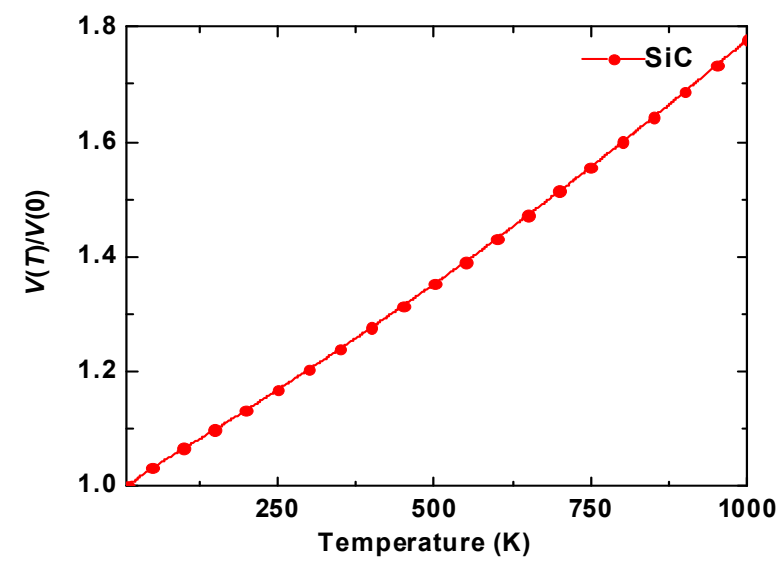

Fig. 21 Variation of the normalized volume $V(T) / V(0)$ with temperature.

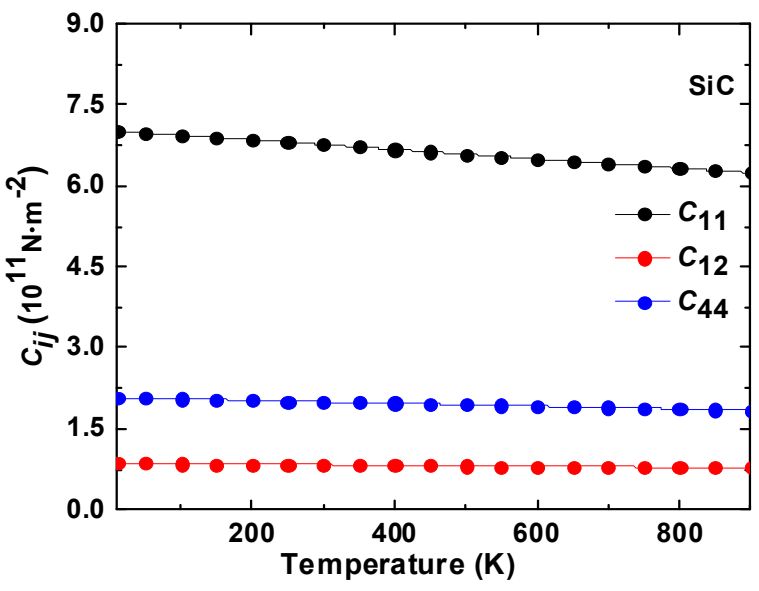

Fig. 22 Variation of aggregate second-order elastic constants with temperature.

linearly with temperature in $\mathrm{ZnS}$ phase. However, aggregate elastic constants $C_{i j}(T)$ increase with pressure as depicted earlier in Fig. 3. It is noticed that (a) the value of $C_{11}$ decreases more steeply with enhancing temperature while $C_{12}$ and $C_{44}$ are less sensitive to temperature for $\mathrm{SiC}$ compound, (b) $C_{11}$ is remarkably larger than $C_{12}$ and $C_{44}$, and (c) values of all aggregate elastic constants $C_{i j}$ are influenced by temperature indicating that anharmonicity is substantial.

The variations of adiabatic bulk modulus $B_{\mathrm{T}}$ and tetragonal modulus $C s$ with temperature $T$ are plotted in Fig. 23. These are compared with the available data [68] at room temperature and are illustrated in Table 2. As expected, both bulk and tetragonal moduli decrease linearly with temperature in $\mathrm{ZnS}$ phase. It should be noted that the second-order elastic constants also depend upon temperature and pressure leading to $C_{12}-$ $C_{44} \neq 0$. Henceforth, B3 phase of $\mathrm{SiC}$ is not only

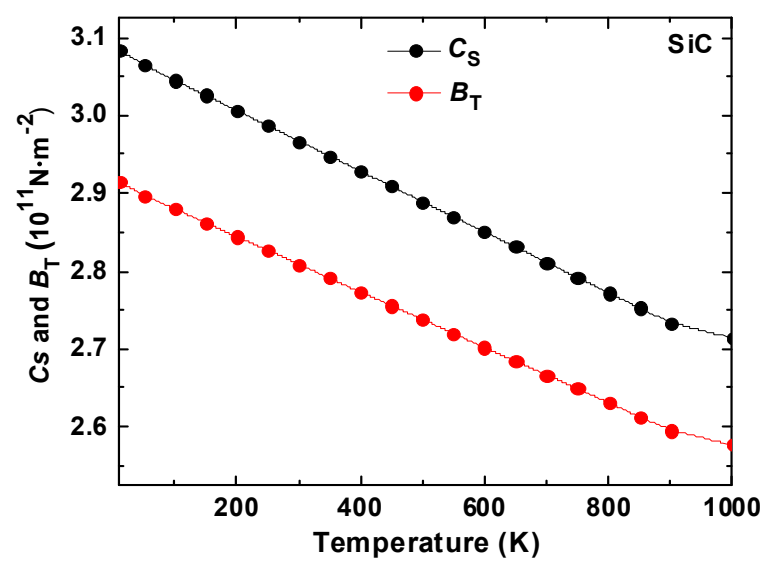

Fig. 23 Variation of aggregate adiabatic bulk modulus $B_{\mathrm{T}}$ and tetragonal modulus $C s$ with temperature. 
mechanically stable but also thermally stable. It is inferred that the aggregate elastic constants $C_{i j}(T)$ are influenced by the incorporation of secondnearest-neighbour interaction as well as sensitive to the short range interactions. Henceforth, the long range forces as Coulomb, charge transfer interaction, and covalency effect are effective. Thus, temperature dependence of aggregate second-order elastic constants can have a direct means to understand the anharmonic effects and coupling coefficients at high temperature explicitly and a balance between anharmonic effects and short range forces.

Usually, the high temperature study of materials leads to an understanding of vibrational anharmonicity that is associated with the interatomic repulsive forces, thermal softening (hardening), and the performance of a material, such as elasticity, extensibility, acoustic transmission velocity, Debye temperature, specific heat, and thermal conductivity at ambient pressure. The high temperature investigations cause laboratory difficulties, and structural changes make the phenomenon more amenable to interpretation. We discuss in Fig. 24 the isotropic shear modulus $G_{\mathrm{H}}$, Voigt's shear modulus $G_{\mathrm{V}}$, and Reuss's shear modulus $G_{\mathrm{R}}$ for $\mathrm{SiC}$ as a function of temperature (at zero pressure). We note that the isotropic shear modulus of $\mathrm{SiC}$ is decreasing with increase in temperature. The steep decrease of $G_{\mathrm{H}}$, $G_{\mathrm{V}}$, and $G_{\mathrm{R}}$ is consistent with aggregate SOEC behavior with temperature.

The temperature dependence of Young's modulus $E$ of $\mathrm{SiC}$ is illustrated in Fig. 25. A decreasing trend is inferred with increase in temperature. Matsumoto and researchers [69] have reported the Young's modulus

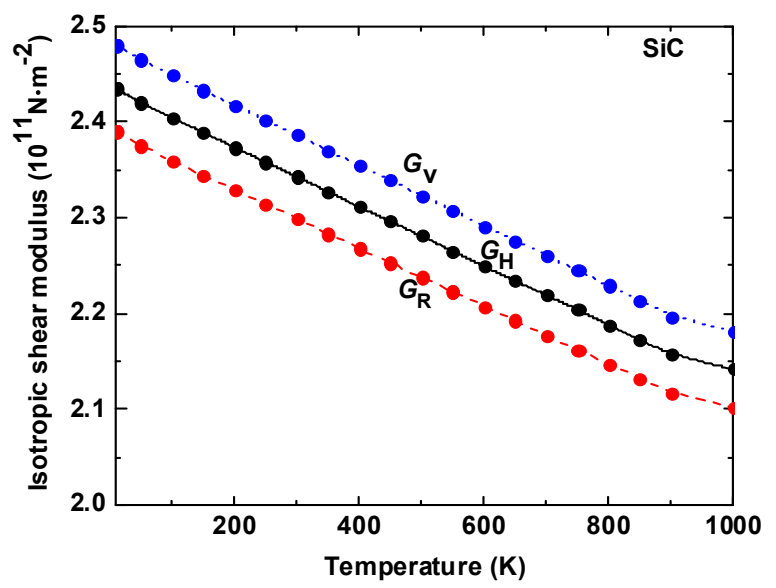

Fig. 24 Variation of isotropic shear moduli $G_{\mathrm{H}}, G_{\mathrm{V}}$, and $G_{\mathrm{R}}$ with temperature.

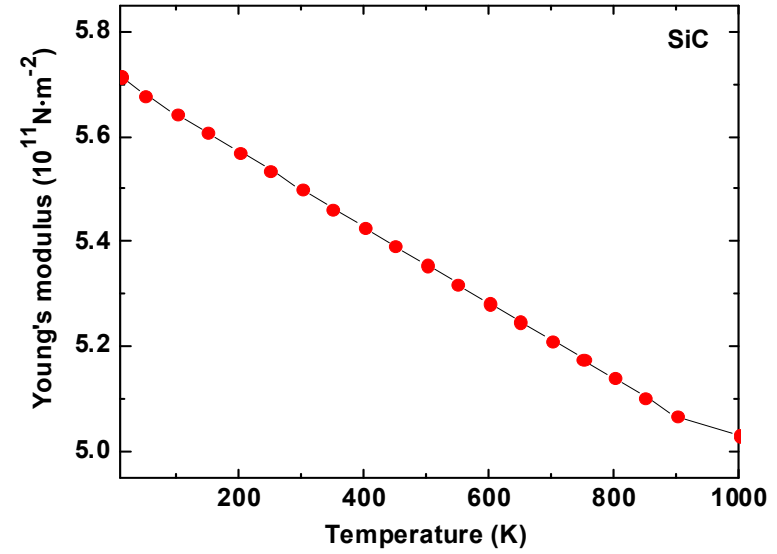

Fig. 25 Variation of Young's modulus $E$ with temperature.

and Poisson's ratio of $\mathrm{SiC}$ at temperatures $>1400{ }^{\circ} \mathrm{C}$ using laser ultrasonics coupled with Fabry-Pérot interferometery as well as ultrasonic pulse method. It is shown that at $T=273 \mathrm{~K}, E$ is about $438 \mathrm{GPa}$ and shows a decreasing behavior with increasing temperature. The calculation presented here leads to a value of about $549 \mathrm{GPa}$ which is comparable to the measured value of $437 \mathrm{GPa}$ at room temperature [69]. Figure 26 illustrates the Poisson's ratio $v$ as a function of temperature. The Poisson's ratio $v$ is independent of temperature and is consistent with the earlier measured $v$ by laser ultrasonics method [69]. We note that the deduced value of $v \approx 0.174$ which is smaller than 0.33 , again indicates that $\mathrm{SiC}$ is brittle in nature. The temperature dependent behavior of Lamé's constants $(\lambda, \mu)$, for $\mathrm{SiC}$ is illustrated in Fig. 27. It is noticed that both Lamé's constants $(\lambda, \mu)$ are decreasing with increasing temperature.

Figure 28 represents the temperature dependence of

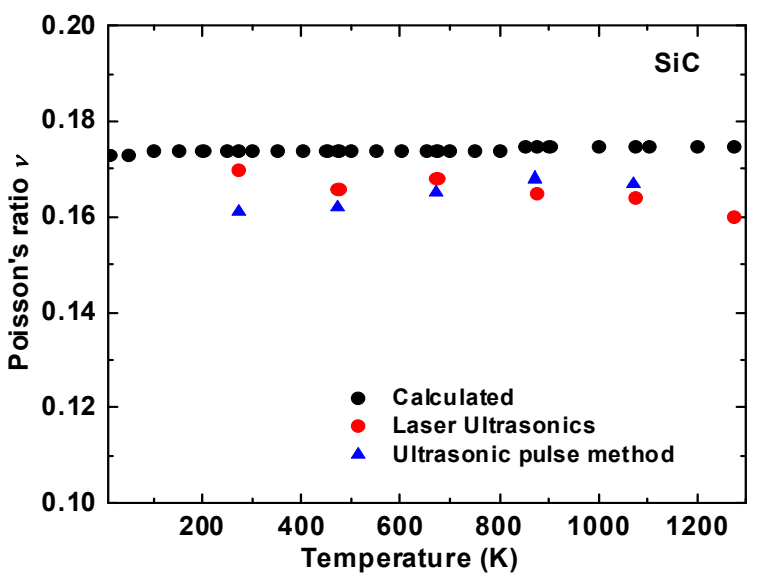

Fig. 26 Variation of Poisson's ratio $v$ with temperature. 


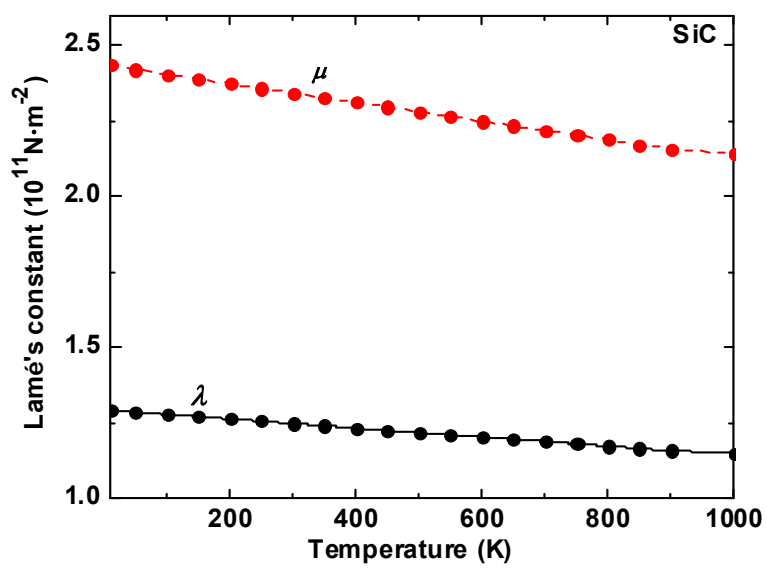

Fig. 27 Variation of Lamé's constants $(\lambda, \mu)$ with temperature.

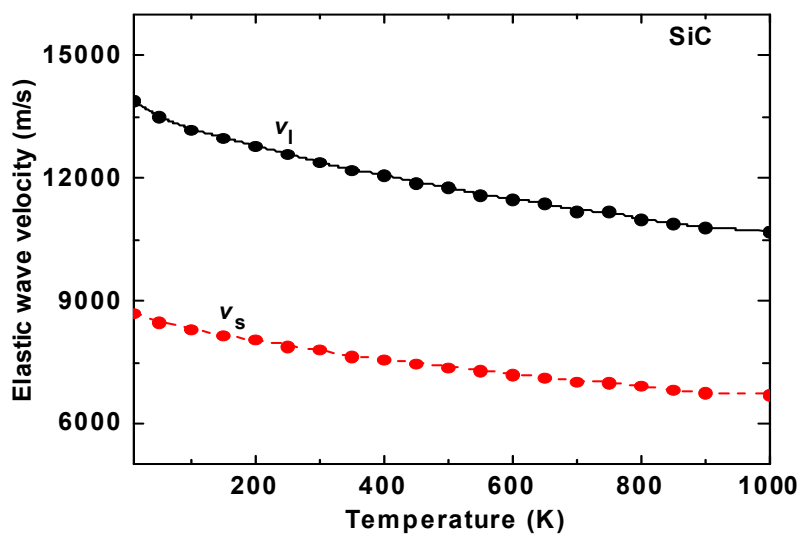

Fig. 28 Temperature dependence of elastic wave velocities $v_{1}$ and $v_{\mathrm{s}}$.

the longitudinal (shear) velocity. We note that both $v_{1}$ and $v_{\mathrm{s}}$ decrease with enhanced temperature in B3 phase. However, $v_{1}$ increases and $v_{\mathrm{s}}$ decreases with enhanced pressure in $\mathrm{B} 3$ phase. Also, both $v_{1}$ and $v_{\mathrm{s}}$ increase with enhanced pressure in $\mathrm{B} 1$ phase of $\mathrm{SiC}$ as inferred in Fig. 14. The values of the longitudinal, shear, and average elastic wave velocities propagating in $\mathrm{SiC}$ compound are documented in Table 2 at room temperature. Deduced values of wave velocities are in good agreement with the measured values by laser ultrasonics method [69]. The high temperature behavior of aggregate elastic constants and others for $\mathrm{SiC}$ can be considered as a predictive study as they cannot be compared due to unavailability of high temperature data. However, deduced Poisson's ratio and its temperature dependence are consistent with the measured Poisson's ratio by laser ultrasonics assisted with Fabry-Pérot interferometery, validating the results obtained by model calculations. The temperature dependent stress studies of ceramics and composites are of vital interest and the results deduced may be useful for future experiments.

Figure 29 represents the variation of Debye temperature as a function of temperature (at zero pressure). Knowledge of Debye temperature of a material not only provides essential features of the vibrational spectrum but is also mandatory for technological and engineering applications, and those results are predictions and seem likely to be useful as a reference for future experimental work. We note that $\theta_{\mathrm{D}}$ decreases rapidly with increasing temperature. Earlier, we have noticed that $\theta_{\mathrm{D}}$ increases with enhanced pressure at zero temperature. The high pressure and high temperature Debye temperature characteristics lead us to comment that the pressure dependent Debye temperature in B3 phase infers the mechanical stiffened bulk modulus due to $\mathrm{Si}-\mathrm{Si}, \mathrm{C}-\mathrm{C}$, and $\mathrm{Si}-\mathrm{C}$ bond compression and bond strengthening due to lattice vibration and also the thermal softening of bulk modulus results from bond expansion and bond weakening due to thermal stress in SiC.

Silicon carbide is obtained by electromelting high purity silica sand with petroleum coke, also of good quality. This melting takes place at high temperature about $2200{ }^{\circ} \mathrm{C}$ and requires a large quantity of energy to produce. It also requires energy for it to dissociate into about $2 / 3 \mathrm{Si}$ and $1 / 3 \mathrm{C}$ in the induction furnace [1]. The better quality raw materials produce better quality $\mathrm{SiC}$, which is lower in nitrogen, sulphur, hydrogen, and other trace elements. Figure 30 shows the temperature dependence of the melting temperature for $\mathrm{SiC}$ estimated from $C_{11}$ elastic constant as discussed

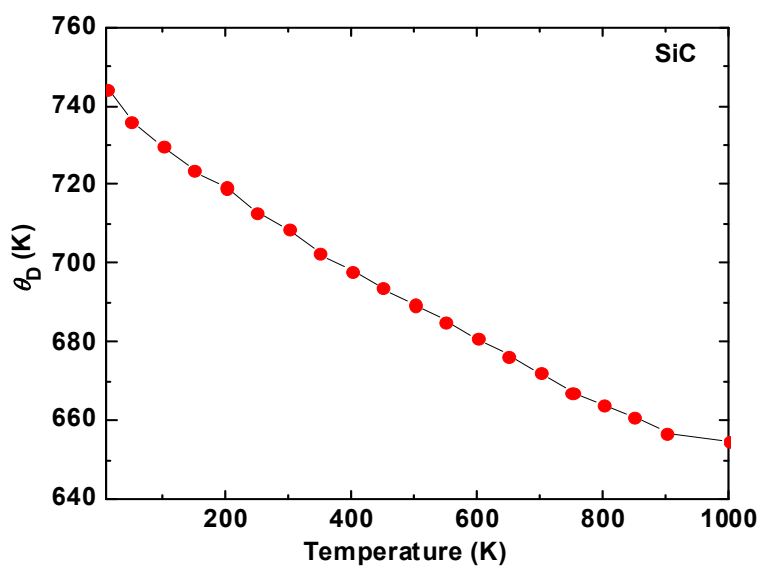

Fig. 29 Debye temperature $\theta_{\mathrm{D}}$ as a function of temperature. 


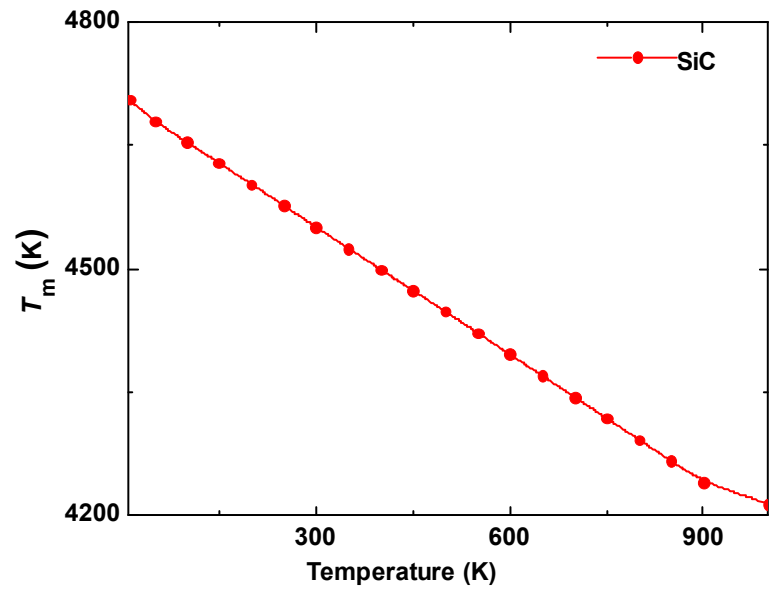

Fig. 30 Variation of melting temperature $T_{\mathrm{m}}$ with temperature.

previously. It is noticed that $T_{\mathrm{m}}$ is suppressed with increased temperature, indicating that there is a decrease in the resistance to deformation by a stress induced due to temperature.

The suppressed $T_{\mathrm{m}}$ infers the weakening of the lattice resulting in thermal softening. Usually, $\mathrm{SiC}$ does not melt; it actually dissolves since its melting point is about $2700{ }^{\circ} \mathrm{C}$. Its behavior in the molten metal is similar to sugar dissolving in coffee. This aspect is very important for the use of $\mathrm{SiC}$. Figure 31 shows Vickers hardness as a function of temperature for SiC. It is clear from the plot that the Vickers hardness $H_{\mathrm{V}}$ decreases in $\mathrm{B} 1$ phase, which indicates that $\mathrm{SiC}$ gets softened with enhanced temperature. Apart from the elastic anisotropy of crystals, the hardness is important to discuss their properties because it is highly correlated with the possibility of inducing microcracks in materials.

Within the framework of quasi-harmonic Debye

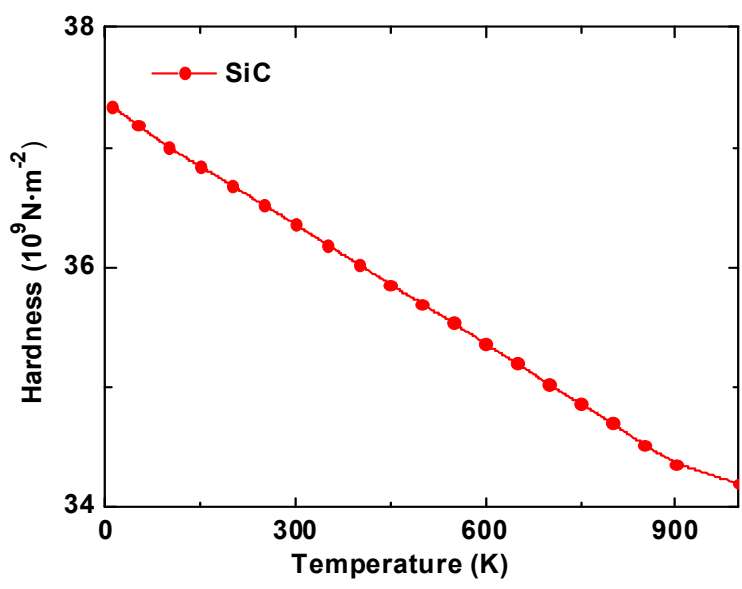

Fig. 31 Variation of hardness $H_{\mathrm{V}}$ with temperature. model, the heat capacity at constant volume $C_{V}$ behavior as a function of temperature for various pressures $(0,50,100$, and $150 \mathrm{GPa})$ is documented in Fig. 32 for SiC. It is inferred from the plots that below room temperature $(300 \mathrm{~K}), C_{V}$ increases very rapidly with temperature at all pressures. Above $300 \mathrm{~K}, C_{V}$ increases slowly with temperature and it almost approaches a constant ideal gas limit-the Dulong-Petit limit $C_{V}(T)=6 R(49.86 \mathrm{~J} /(\mathrm{mol} \cdot \mathrm{K}))$ at higher temperatures as well as at all pressures for all compounds. These are consistent with earler results on thermodynamical properties using LDA [70,71]. Further, we have sketched the variations of the thermal expansion $\alpha_{\text {th.exp }}$ with temperature at various pressures illustrated in Fig. 33 for SiC. Furthermore, at low temperatures, $\alpha_{\text {th.exp }}$ enhances rapidly with temperature at zero pressure. The slope of $\alpha_{\text {th.exp }}$ gradually decreases at higher temperatures at all pressures except at $P=0$. It should be noted that the

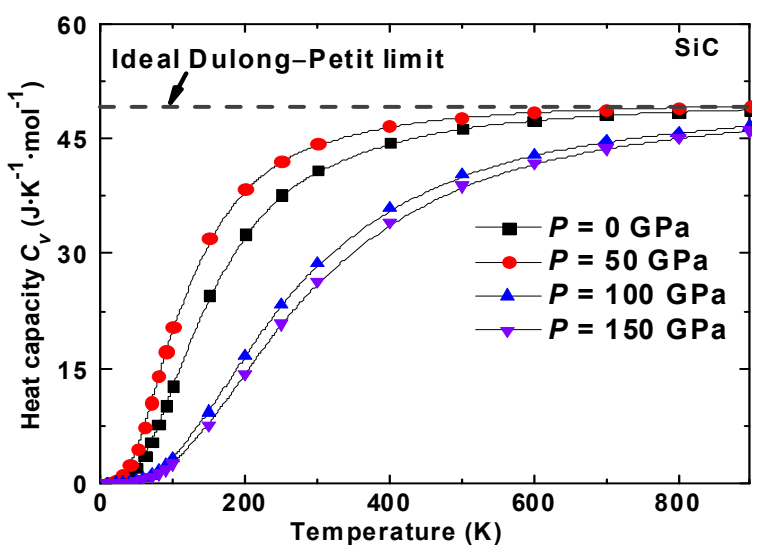

Fig. 32 Variation of heat capacity $C_{V}$ with temperature at different pressures.

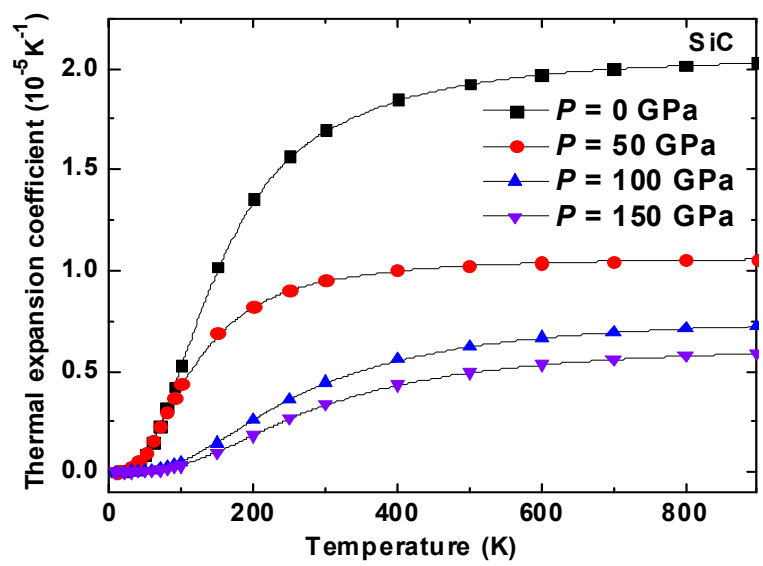

Fig. 33 Variation of thermal expansion coefficient with temperature at different pressures. 
thermal expansion coefficient $\alpha_{\text {th.exp }}$ decreases with the increase of pressure.

\section{Conclusions}

The present study thus addresses for the first time, the high pressure and high temperature dependent aggregate second-order elastic constants, Cauchy discrepancy, anisotropy in higher order elastic constants, ductile nature, propagation of compression and shear wave, Grüneisen parameter, Debye temperature, melting temperature, hardness, heat capacity, and thermal expansion coefficient of $\mathrm{SiC}$ by formulating an effective interatomic interaction potential with emphasis on long range Coulomb, charge transfer, covalency, and zero point energy effect as well as short range overlap repulsion extended up to the second neighbor ions and van der Waals interaction effect. Deduced values of free parameters allow us to predict phase transition pressure and associated volume collapse. We have found volume discontinuity in pressure volume phase diagram, identifying structural phase transition from $\mathrm{ZnS}$ (B3) to $\mathrm{NaCl}$ (B1) structure for $\mathrm{SiC}$.

From the computed values of pressure dependent Pugh's ratio $\phi=B_{\mathrm{T}} / G_{\mathrm{H}}$, Poisson's ratio $v$, and the Cauchy pressure $C_{12}-C_{44}$, we suggest that $\mathrm{SiC}$ ceramic shows brittle nature at low and high pressures. High temperature Poisson's ratio $v$ of about 0.174 of $\mathrm{SiC}$ ceramic infers its brittle nature, and temperature dependent behavior infers a decreasing trend consistent with the earlier measured value by laser ultrasonics method. Furthermore, compression and shear wave velocities are in good agreement with the measured values using laser ultrasonics method.

The pressure dependent isothermal compressibility in $\mathrm{SiC}$ infers that the lattice is stiffened with increase in pressure in $\mathrm{B} 3$ phase, gets softened at phase transition pressure and again becomes stiff at higher pressures, i.e., in $\mathrm{B} 1$ phase. The mechanical stiffened bulk modulus in $\mathrm{SiC}$ is attributed to $\mathrm{Si}-\mathrm{Si}, \mathrm{C}-\mathrm{C}$, and $\mathrm{Si}-\mathrm{C}$ bond compression and bond strengthening due to lattice vibration in both B3 and B1 phases. The compressibility at zero pressure is reduced by about $1 \%$ of that at transition pressure in $\mathrm{B} 3$ phase and the magnitude of $\beta$ at transition pressure is about $0.3 \%$ of that at higher pressures ( 150 GPa) leading to giant lattice softening noticed at the boundary of B3-B1 phase transition in SiC. The temperature dependent adiabatic bulk modulus in $\mathrm{SiC}$ ceramic documents a decreasing trend with enhanced temperature in $\mathrm{ZnS}$ phase. The outcome is the thermal softening of adiabatic bulk modulus resulting from $\mathrm{Si}-\mathrm{Si}, \mathrm{C}-\mathrm{C}$, and $\mathrm{Si}-\mathrm{C}$ bond expansion and bond weakening due to thermal stress in $\mathrm{SiC}$.

The aggregate elastic constants $C_{i j}(T)$ decrease linearly with temperature in $\mathrm{ZnS}$ phase implying the importance of the long range forces as Coulomb and charge transfer interaction in $\mathrm{SiC}$ ceramic. The pressure dependent thermal expansion coefficient $\alpha_{\text {th.exp }}$ decreases nonlinearly with pressure for both $\mathrm{ZnS}$ and $\mathrm{NaCl}$ phases. The discontinuity at $P_{\mathrm{T}}$ in $\alpha_{\text {th.exp }}$ confirms the mechanical softening in $3 \mathrm{C} \mathrm{SiC}$ ceramic. However, the temperature dependent thermal expansion coefficient $\alpha_{\text {th.exp }}$ increases rapidly (nonlinearly) at low temperatures and remains independent of temperature away from room temperature at all pressures. Also, $\alpha_{\text {th.exp }}$ suppresses with enhanced pressures at higher temperatures.

Within the framework of quasi-harmonic Debye model, the heat capacity at different temperatures decreases nonlinearly with the applied pressures. It infers that the vibration frequency of the particles in $3 \mathrm{C} \mathrm{SiC} \mathrm{ceramic} \mathrm{changes} \mathrm{with} \mathrm{pressure} \mathrm{as} \mathrm{well} \mathrm{as}$ temperature. For higher temperatures $T \rightarrow \theta_{\mathrm{D}}$, the variation in heat capacity with pressure is weak and at $P_{\mathrm{T}}$. The temperature dependent heat capacity $C_{V}$ increases very rapidly with increase in temperature at all pressures for $T<300 \mathrm{~K}$. Furthermore, $C_{V}(T)$ is consistent with ideal Dulong-Petit limit of $3 R$, at higher temperatures as well as at all pressures for $\mathrm{SiC}$ ceramic which cannot be compared due to unavailability of data. To our knowledge, these are the first quantitative theoretical prediction of the high pressure and high temperature dependent mechanical, elastic, thermal, and thermodynamical properties of $\mathrm{SiC}$ ceramic addressing mechanical stiffening, thermal softening, and ductile nature and still await experimental confirmations.

\section{References}

[1] Levinshtein ME, Rumyantsev SL, Shur MS. Properties of Advanced Semiconductor Materials: GaN, AlN, InN, BN, $\mathrm{SiC}, \mathrm{SiGe}$. New York: Wiley, 2001.

[2] Parfenova II. Substitutional $3 d$ impurities in cubic silicon 
carbide. Semiconductors 2004, 38: 189-191.

[3] Yoshida M, Onodera A, Ueno M, et al. Pressure-induced phase transition in SiC. Phys Rev B 1993, 48: 10587(R).

[4] Sekine T, Kobayashi T. Shock compression of $6 \mathrm{H}$ polytype SiC to 160 GPa. Phys Rev B 1997, 55: 8034.

[5] Chang KJ, Cohen ML. Ab initio pseudopotential study of structural and high-pressure properties of SiC. Phys Rev B 1987, 35: 8196.

[6] Cheong BH, Chang KJ, Cohen ML. Pressure dependences of band gaps and optical-phonon frequency in cubic SiC. Phys Rev B 1991, 44: 1053.

[7] Karch K, Bechstedt F, Pavone P, et al. Pressure-dependent properties of SiC polytypes. Phys Rev B 1996, 53: 13400.

[8] Miao MS, Lambrecht WRL. Unified path for high-pressure transitions of $\mathrm{SiC}$ polytypes to the rocksalt structure. Phys Rev B 2003, 68: 092103.

[9] Miao MS, Prikhodko M, Lambrecht WRL. Changes of the geometry and band structure of $\mathrm{SiC}$ along the orthorhombic high-pressure transition path between the zinc-blende and rocksalt structures. Phys Rev B 2002, 66: 064107.

[10] Miao MS, Prikhodko M, Lambrecht WRL. Comment on "orthorhombic intermediate state in the zinc blende to rocksalt transformation path of $\mathrm{SiC}$ at high pressure". Phys Rev Lett 2002, 88: 189601.

[11] Durandurdu M, Drabold DA. Ab initio simulation of high-pressure phases of GaAs. Phys Rev B 2002, 66: 045209.

[12] Durandurdu M. Pressure-induced phase transition of SiC. J Phys: Condens Matter 2004, 16: 4411.

[13] Shimojo F, Ebbsjö I, Kalia RK, et al. Molecular dynamics simulation of structural transformation in silicon carbide under pressure. Phys Rev Lett 2000, 84: 3338.

[14] Lu, Y-P, He D-W, Zhu J, et al. First-principles study of pressure-induced phase transition in silicon carbide. Physica B 2008, 403: 3543-3546.

[15] Vashishta P, Kalia RK, Nakano A, et al. Interaction potential for silicon carbide: A molecular dynamics study of elastic constants and vibrational density of states for crystalline and amorphous silicon carbide. J Appl Phys 2007, 101: 103515.

[16] Stillinger FH, Weber TA. Computer simulation of local order in condensed phases of silicon. Phys Rev B 1985, 31: 5262.

[17] Oganov AR. Modern Methods of Crystal Structure Prediction. Weinheim, Germany: Wiley-VCH Verlag GmbH \& Co. KGaA, 2010.

[18] Dion M, Rydberg H, Schröder E, et al. Van der Waals density functional for general geometries. Phys Rev Lett 2004, 92: 246401.

[19] Varshney D, Shriya S. Elastic, mechanical and thermodynamic properties at high pressures and temperatures of transition metal monocarbides. Int $J$ Refract Met H 2013, 41: 375-401.

[20] Hafemeister DW, Flygare WH. Outer-shell overlap integrals as a function of distance for halogen-halogen, halogen-alkali, and alkali-alkali ions in the alkali halide lattices. J Chem Phys 1965, 43: 795.
[21] Tosi MP. Cohesion of ionic solids in the Born model. Solid State Phys 1964, 16: 1-120.

[22] Born M, Huang K. Dynamical Theory of Crystal Lattices. Oxford: Clarendon Press, 1956.

[23] Motida K. Szigeti charge and its correlation with hyperfine coupling constant of doped $\mathrm{Mn}^{2+}$ ion in divalent metal compounds. J Phys Soc Jpn 1980, 49: 213-217.

[24] Motida K. Effect of covalency on phonon dispersion relations in $\mathrm{NaCl}$ type alkali halide crystals. J Phys Soc Jpn 1986, 55: 1636-1649.

[25] Slater JC, Kirkwood JG. The van der Waals forces in gases. Phys Rev 1931, 37: 682.

[26] Löwdin P-O. Quantum theory of cohesive properties of solids. Adv Phys 1956, 5: 1-171.

[27] Singh RK. Many body interactions in binary ionic solids. Phys Rep 1982, 85: 259-401.

[28] Varshney D, Sharma P, Kaurav N, et al. Study of elastic properties and their pressure dependence of semi magnetic semiconductors. J Phys Soc Jpn 2005, 74: 382-388.

[29] Tessman JR, Kahn AKH, Shockley W. Electronic polarizabilities of ion in crystals. Phys Rev 1953, 92: 890.

[30] Shannon RD. Dielectric polarizabilities of ions in oxides and fluorides. J Appl Phys 1993, 73: 348.

[31] Weast RC. Handbook of Chemistry and Physics, 63rd edn. BocaRaton, FL, USA: CRC Press, 1982.

[32] Aleksandrov IV, Goncharov AF, Stishov SM, et al. Equation of state and Raman scattering in cubic $\mathrm{BN}$ and SiC at high pressures. JETP Lett 1989, 50: 127-131.

[33] Feldman DW, Parker JH, Choyke Jr. WJ, et al. Phonon dispersion curves by Raman scattering in $\mathrm{SiC}$, polytypes 3C, 4H, 6H, 15R, and 21R. Phys Rev 1968, 173: 787.

[34] Liu J, Vohra YK. Raman modes of $6 \mathrm{H}$ polytype of silicon carbide to ultrahigh pressures: A comparison with silicon and diamond. Phys Rev Lett 1994, 72: 4105.

[35] Wagman DD, Evans WH, Parker VB, et al. Selected values of chemical thermodynamics properties. Tables for the first thirty-four elements in the standard order of arrangement. Technical Note 270-3. U.S. Department of Commerce. Natioanl Bureau of Standards. 1968.

[36] Lambrecht WRL, Segall B, Methfessel M, et al. Calculated elastic constants and deformation potentials of cubic SiC. Phys Rev B 1991, 44: 3685.

[37] Murnaghan FD. The compressibility of media under extreme pressures. PNAS 1944, 30: 244-247.

[38] Guo Y-D, Yang Z-J, Gao Q-H, et al. The phase transition, and elastic and thermodynamic properties of $\mathrm{CaS}$ derived from first-principles calculations. $J$ Phys: Condens Matter 2008, 20: 115203.

[39] Denteneer PJH, van Haeringen W. Ground-state properties of polytypes of silicon carbide. Phys Rev B 1986, 33: 2831.

[40] Tolpygo KB. Optical, elastic and piezoelectric properties of ionic and valence crystals with ZnS-type lattice. Sov Phys Solid State 1961, 2: 2367-2376.

[41] Arlt G, Schodder GR. Some elastic constants of silicon carbide. J Acoust Soc Am 1965, 37: 384.

[42] Lee DH, Joannopoulos JD. Simple scheme for deriving atomic force constants: Application to SiC. Phys Rev Lett 
1982, 48: 1846.

[43] Yean DH, Riter Jr. JR. Estimates of isothermal bulk moduli for group iva crystals with the zincblende structure. $J$ Phys Chem Solids 1971, 32: 653-655.

[44] Carnahan RD. Elastic properties of silicon carbide. $J \mathrm{Am}$ Ceram Soc 1968, 51: 223-224.

[45] Churcher N, Kunc K, Heine V. Calculated ground-state properties of silicon carbide. J Phys C: Solid State Phys 1986, 19: 4413.

[46] Gao F, Weber WJ. Mechanical properties and elastic constants due to damage accumulation and amorphization in SiC. Phys Rev B 2004, 69: 224108.

[47] Tang M, Yip S. Lattice instability in $\beta$-SiC and simulation of brittle fracture. J Appl Phys 1994, 76: 2719.

[48] Vukcevich MR. On the stability of the alkali halide lattices under hydrostatic pressure. Phys Status Solidi b 1972, 54: 435-440.

[49] Barsch GR. Relation between third-order elastic constants of single crystals and polycrystals. J Appl Phys 1968, 39: 3780.

[50] Hill R. The elastic behaviour of a crystalline aggregate. Proc Phys Soc A 1952, 65: 349.

[51] Voigt W. Handbook of Crystal Physics. Leipzig: Teubner, 1928.

[52] Reuss A. Berechnung der Fließgrenze von Mischkristallen auf Grund der Plastizitätsbedingung für Einkristalle. ZAMM-Journal of Applied Mathematics and Mechanics / Zeitschrift für Angewandte Mathematik und Mechanik 1929, 9: 49-58.

[53] Pugh SF. Relations between the elastic moduli and the plastic properties of polycrystalline pure metals. The London, Edinburgh, and Dublin Philosophical Magazine and Journal of Science: Series 7 1954, 45: 823-843.

[54] Frantsevich N, Voronov FF, Bokuta SA. In Elastic Constants and Elastic Moduli of Metals and Insulators Handbook. Frantsevich IN, Ed. Kiev: Naukova Dumka, 1983: 60-180.

[55] Goel S, Luo X, Reuben RL, et al. Atomistic aspects of ductile responses of cubic silicon carbide during nanometric cutting. Nanoscale Res Lett 2011, 6: 589.

[56] Patten J, Gao W, Yasuto K. Ductile regime nanomachining of single-crystal silicon carbide. J Manuf Sci Eng 2004, 127: 522-532.

[57] Schreiber E, Anderson OL, Soga N. Elastic Constants and Their Measurements. New York: McGraw-Hill, 1973: $56-57$.

[58] Bouhemadou A, Khenata R, Kharoubi M, et al. FP-APW + lo calculations of the elastic properties in zinc-blende III-P compounds under pressure effects. Comput Mater Sci 2009, 45: 474-479.

[59] Maachou A, Aboura H, Amrani B, et al. Structural stabilities, elastic and thermodynamic properties of Scandium Chalcogenides via first-principles calculations. Comput Mater Sci 2011, 50: 3123-3130.

[60] Kleinman L. Deformation potentials in silicon. I. Uniaxial strain. Phys Rev 1962, 128: 2614.

[61] Kim K, Lambrecht WRL, Segal B. Electronic structure of GaN with strain and phonon distortions. Phys Rev B 1994, 50: 1502.

[62] Blackman M. Contributions to the theory of the specific heat of crystals. I. Lattice theory and continuum theory. Proceedings of the Royal Society of London. Series A, Mathematical and Physical Sciences 1935, 148: 365-383.

[63] Gopal ESR. Specific Heats at Low Temperatures. New York: Plenum Press, 1966.

[64] Tari A. The Specific Heat of Matter at Low Temperatures. London: Imperial College Press, 2003.

[65] Goel P, Choudhury N, Chaplot SL. Superionic behavior of lithium oxide $\mathrm{Li}_{2} \mathrm{O}$ : A lattice dynamics and molecular dynamics study. Phys Rev B 2004, 70: 174307.

[66] $\mathrm{Hu} \mathrm{YH}$, Ruckenstein E. Highly effective $\mathrm{Li}_{2} \mathrm{O} / \mathrm{Li}_{3} \mathrm{~N}$ with ultrafast kinetics for $\mathrm{H}_{2}$ storage. Ind Eng Chem Res 2004, 43: $2464-2467$.

[67] Li X-F, Chen X-R, Meng C-M, et al. Ab initio calculations of elastic constants and thermodynamic properties of $\mathrm{Li}_{2} \mathrm{O}$ for high temperatures and pressures. Solid State Commun 2006, 139: 197-200.

[68] Kunc K, Balkanski M, Nusimovici MA. Lattice dynamics of several $\mathrm{A}^{N} \mathrm{~B}^{8-N}$ compounds having the zincblende structure. II. Numerical calculations. Phys Status Solidi $b$ 1975, 72: 229-248.

[69] Matsumoto T, Nose T, Nagata Y, et al. Measurement of high-temperature elastic properties of ceramics using a laser ultrasonic method. $J$ Am Ceram Soc 2001, 84: $1521-1525$.

[70] Zhang X, Quan S, Ying C, et al. Theoretical investigations on the structural, lattice dynamical and thermodynamic properties of XC $(\mathrm{X}=\mathrm{Si}, \mathrm{Ge}, \mathrm{Sn})$. Solid State Commun 2011, 151: 1545-1549.

[71] Zhang X-D, Cui S-X, Shi H-F. Theoretical study of thermodynamics properties and bulk modulus of $\mathrm{SiC}$ under high pressure and temperature. Chinese Phys Lett 2014, 31: 016401 .

Open Access The articles published in this journal are distributed under the terms of the Creative Commons Attribution 4.0 International License (http://creativecommons. org/licenses/by/4.0/), which permits unrestricted use, distribution, and reproduction in any medium, provided you give appropriate credit to the original author(s) and the source, provide a link to the Creative Commons license, and indicate if changes were made. 